\title{
The Schwarz Alternating Method in Solid Mechanics
}

\author{
Alejandro Mota ${ }^{1 *}$ Irina Tezaur ${ }^{2}$, Coleman Alleman ${ }^{1}$ \\ ${ }^{1}$ Mechanics of Materials Department \\ Sandia National Laboratories \\ Livermore CA 94550, USA \\ ${ }^{2}$ Extreme Scale Data Science \& Analytics \\ Sandia National Laboratories \\ Livermore CA 94550, USA
}

January 31,2017

\begin{abstract}
We advance the Schwarz alternating method as a means for concurrent multiscale coupling in finite deformation solid mechanics. We prove that the Schwarz alternating method converges to the solution of the problem on the entire domain and that the convergence rate is geometric provided that each of the subdomain problems is well-posed, i.e. their corresponding energy density functions are quasi-convex. It is shown that the use of a Newton-type method for the solution of the resultant nonlinear system leads to two kinds of block linearized systems, depending on the treatment of the Dirichlet boundary conditions. The first kind is a symmetric block-diagonal linear system in which each diagonal block is the tangent stiffness of each subdomain, i.e. the off-diagonal blocks are all zero and the coupling terms appear only on the right-hand side. The second kind is a nonsymmetric block system with off-diagonal coupling terms. Several variants of the Schwarz alternating method are proposed for the first kind of linear system, including one in which the Schwarz alternating iterations and the Newton iterations are combined into a single scheme. This version of the method is particularly attractive, as it lends itself to a minimally intrusive implementation into existing finite element codes. Finally, we demonstrate the performance of the proposed variants of the Schwarz alternating method on several one-dimensional and three-dimensional examples.
\end{abstract}

Keywords: Schwarz alternating method, concurrent multiscale coupling, finite deformation, variational methods

\section{Introduction}

Materials such as metals, metal alloys, sands, soils, and others develop regions of concentrated strain in narrow bands when subject to certain loading conditions. This strain localization phenomenon occurs at the

*Email: amota@sandia.gov

(C) 2016. This manuscript version is made available under the Elsevier user license http://www.elsevier.com/open-access/userlicense/1.0/ 
micron scale and may lead to the eventual failure of systems and structures. For this reason, strain localization is of critical significance in engineering applications [66, 14, 3, 51, 70, 27].

It is not feasible to conduct micro-scale simulations for macroscopic problems to fully resolve the strain localization phenomenon described above. So that computational resources can be efficiently allocated, it is advantageous to perform multiscale analysis. A fine scale model is used in regions to resolve fields that lead to strain localization. Away from these regions, a less expensive coarse scale model is used to capture the far field behavior [9, 28, 6, 49, 47].

The multiscale methods used to incorporate fine scale mechanical behavior into macroscopic analysis can be broadly classified into three categories [1].

The first class of methods model the inherent multiscale character and introduce a length scale through phenomenological laws. For instance, Fleck and Hutchinson [23] introduce a strain gradient into constitutive models such that multiple material length parameters can be defined for the field equations corresponding to different dominant mechanisms. For problems where the deformation band thickness is much smaller than the mesh size, enhancement basis functions or localization elements can be inserted to idealize the deformation band with embedded strongly or weakly discontinuous displacement fields [11, 12, 69]. We refer to these as sub-grid methods.

The second class of methods incorporate micro-structural information from unit cells to compute effective macroscopic properties on the coarse domain [22, 8]. By passing or up-scaling stress and internal variables, this type of approach establishes an effective macroscale constitutive relation for the coarse domain, and it introduces a length scale that regularizes the governing equation. Kouznetsova, Geers, and Brekelmans [34] introduce a gradient-enhanced homogenization scheme in which the macroscopic stress, strain, and their gradients are obtained from solutions of boundary value problems applied on representative volume elements. Recently, a similar unit cell approach has been applied to porous flow problems by White, Borja, and Fredrich [68] and Sun, Andrade, and Rudnicki [60] to conduct large scale flow simulations on tomographic images. In both cases, the quality of the hierarchical multiscale analysis depends on the existence of a representative elementary volume and the design of sequential coupling schemes that establish information exchange across scales. We designate these as homogeneization methods.

In the third class of methods, computational efforts are concentrated in the region where strain localization occurs, once this region is identified. The fine-scale processes in this critical region are represented by appropriate constitutive laws, special numerical methods, or fine meshes. This region is then connected to a coarse region in which a simpler low-cost macroscopic model is used. There are a number of established methods that have been used successfully for these types of coupling: discrete and continuum models (e.g. Bauman et al. [6], Prudhomme et al. [49], Parks, Bochev, and Lehoucq [47], and Bauman, Oden, and Prudhomme [5]), classical local and nonlocal elastic continuum (e.g. Han and Lubineau [31]), structural elements with various mesh refinements (e.g. Ben Dhia and Rateau [10]), and continnum to continuum (e.g. Sun and Mota [61]). We refer to these as concurrent coupling methods. The advantage of concurrent coupling methods is that they are able to effectively allocate computational resources to sub-domains, depending upon the need to characterize their fine-scale behavior. Among the best known concurrent coupling methods are FETI and mortar finite elements [7,21]. FETI is closely related to the variational multiscale method of Garikipati and Hughes [26], and early in our research on coupling methods we investigated the development of a method based on variational multiscale. Ultimately we decided against this due to the complexity of the computer implementation of the involved projection operators, and also due to the introduction of Lagrange mutipliers that require careful selection of function spaces for the formulation to be stable [61]. Likewise, mortar methods also require careful selection of function spaces for the Lagrange multipliers [7]. In addition, the sharp interfaces introduced by mortar methods lead to reflection and refraction artifacts in dynamic applications [28]. 
The objective of this work is to introduce a method that has the following properties:

- it is concurrent in the sense that information flows back and forth between the coupled scales;

- it couples continuum to continuum but can be extended to couple atomistics to continuum;

- it can use different solution methods for each scale;

- it is computationally efficient;

- it readily uses existing finite elements and constitutive models;

- it simplifies the task of building meshes for the different scales;

- and, perhaps most important of all, it averts invasive changes to existing simulation codes.

With respect to these requirements, established methods have a number of shortcomings. Sub-grid methods require the development of special elements or consitutive models. Homogeneization methods are not concurrent, in that the flow of information is unidirectional: kinematic information flows from the coarse to the fine scale, and stress and internal variable information flows from the fine to the coarse scale. Existing concurrent coupling methods satisfy some of these requirements but may be invasive to implement.

Herein, we propose the Schwarz alternating method for concurrent coupling between continuum-based micro-scale and macro-scale finite element simulations for the fully inelastic finite deformation problem. As will become apparent in the subsequent sections, the Schwarz alternating method satisfies most, if not all, of the requirements enumerated above.

\subsection{Previous Work}

The Schwarz alternating method, the earliest known domain decomposition method, was introduced in a seminal paper by Hermann Schwarz in 1870 [55]. Schwarz's motivation for developing the famous algorithm that bears his name was theoretical: he sought to prove the existence of harmonic functions in irregular domains by expressing them as the union of domains of regular shape (e.g., rectangles and circles). It was only much later, after the appearance of computers, that the method was used as a numerical technique for solving partial differential equations. In particular, the method has gained prevalence in the domain decomposition community, where it is typically used as a preconditioner for Krylov iterative methods to solve linear algebraic equations [57, 64, 40, 50]. Although it would perhaps seem natural to use the Schwarz alternating method to couple two problems on a domain that is the union of two overlapping subdomains, there are surprisingly few works in the literature that employ the method to achieve concurrent coupling in this way. In recent years, several authors have used the method for concurrent multiscale coupling of atomistic and continuum scales, e.g. Hadjiconstantinou and Patera [30], Werder, Walther, and Koumoutsakos [67], Parks, Bochev, and Lehoucq [47], and Pandurangan, Li, and Ng [46]. In these works, different numerical schemes are applied in the atomistic and continuum regions, each restricted to its own subdomain, and the transfer of information from the continuum to the atomistic scale occurs in the overlap region. Schwarz-like methods have also been proposed to couple distinct physics in different subdomains, for example, fluid-structure interaction (Engel and Griebel [18]), and computational fluid dynamics with aero-acoustics (Borrel, Halpern, and Ryan [13] and Ryan, Halpern, and Borrel [52]). In these works, the governing equations are solved alternatively in each subdomain, and the necessary information, such as structure-fluid loading, is transferred through boundary conditions at the interface. To the best of our knowledge, the present work is the first to use the Schwarz alternating method to accomplish concurrent multiscale continuum-to-continuum coupling for finite-deformation solid mechanics. 


\subsection{Convergence and Theoretical Properties}

The relevant literature reveals that a number of authors, including Schwarz himself, studied the convergence of the Schwarz alternating method. A critical requirement for the convergence of the method is that the size of the overlap region be greater than zero [57, 64, 40, 50]. The original proof by Schwarz was for linear elliptic second order equations and relied on the maximum principle [55]. Subsequent authors developed other approaches to prove convergence. Variational methods were a popular approach during the 1930s-1960s, employed for linear elasticity by Sobolev [58], for self-adjoint elliptic equations by Mikhlin [41], for polyharmonic functions by Prager [48], and for higher-order linear equations, e.g. biharmonic, by Morgenstern [42] and Babuška [2] as well as numerous others. Many of these works applied variational ideas after recasting the method as a series of successive projections.

Most, if not all, of these works investigate the convergence of the Schwarz alternating method for the specific case of linear elliptic partial differential equations. Beginning in the 1980s, researchers began to examine the convergence of the method when applied to nonlinear problems. A series of authors, including Lions [38], Badea [4], and Lui [39] proved convergence of the method for certain types of nonlinear monotone problems. In this work, we prove that the Schwarz alternating method converges for the finite-deformation solid mechanics problem.

Assuming that the convergence of the Schwarz alternating method for a given problem can be established, a question that arises is whether anything can be said about its convergence rate. It has been shown that the method converges geometrically for linear as well as nonlinear monotone problems [65, 38, 4]. Nevertheless, rigorous estimates of the a priori convergence rate of the method as a function of the size of the overlap region seem to be lacking at the present time. An attempt to derive the theoretical convergence rate of the method for linear elliptic problems was made by Evans et al. [19], Evans et al. [20], and Li-Shan and Evans [37]. There, it was shown analytically as well as numerically that the convergence rate of the Schwarz alternating method increases with the size of the overlap region. In this work, we prove that the method converges geometrically, and we investigate the numerical convergence of the method as a function of overlap size.

Finally, while most of the works cited above present their analysis for the specific case of two subdomains, extension to multiple subdomains is in general straightforward. The case of multiple subdomains is considered specifically in Li-Shan and Evans [37], Lions [38], and Badea [4]. The theoretical results presented in this work can be extended for the coupling of multiple subdomains. We demonstrate such coupling in a numerical example.

\subsection{Scope and Organization}

The remainder of this work is organized as follows. The formulation of the Schwarz alternating method is presented in Section 2. We first introduce the variational formulation of the finite-deformation inelastic solid mechanics problem to set the context and notation in Section 2.1. We then present the variational formulation of the Schwarz alternating method for coupling quasi-static mechanics problems defined on multiple overlapping subdomains in Section 2.2. The linearized problem and two ways to treat Dirichlet boundary conditions for coupling are described in Section 2.3. Four variants of the Schwarz alternating method derived from our formulation are introduced in Section 2.4. In Section 3, we give conditions, in the form of a theorem, under which convergence of the Schwarz alternating method is guaranteed. This result is proven in Appendix A. Section 4 is devoted to numerical experiments which demonstrate the convergence properties and effectiveness of the proposed method. We first discuss our two implementations of the method in Section 4.1. In Section 4.2, we introduce the error measures to be used subsequently. Then several quasi-static mechanics problems are considered: a one-dimensional bar in Section 4.3, a three-dimensional model of a rectangular box in Section 4.4, a three-dimensional model of a notched cylinder in Section 4.5, 
and a three-dimensional model of two metal blocks joined by a laser weld in Section 4.6. Finally, in Section 5, we discuss results, address outstanding issues, outline possible future work, and provide some conclusions.

\section{Formulation of the Schwarz Alternating Method}

We start by defining the standard finite deformation variational formulation to establish notation before presenting the formulation of the coupling method.

\subsection{Variational Formulation on a Single Domain}

Consider a body as the open set $\Omega \subset \mathbb{R}^{3}$ undergoing a motion described by the mapping $x=\varphi(X): \Omega \rightarrow \mathbb{R}^{3}$, $\boldsymbol{X} \in \Omega$. Assume that the boundary of the body is $\partial \Omega=\partial_{\boldsymbol{\varphi}} \Omega \cup \partial_{\boldsymbol{T}} \Omega$ with unit normal $\boldsymbol{N}$, where $\partial_{\boldsymbol{\varphi}} \Omega$ is a displacement boundary, $\partial_{\boldsymbol{T}} \Omega$ is a traction boundary, and $\partial_{\boldsymbol{\varphi}} \Omega \cap \partial_{\boldsymbol{T}} \Omega=\emptyset$. The prescribed boundary displacements or Dirichlet boundary conditions are $\chi: \partial_{\varphi} \Omega \rightarrow \mathbb{R}^{3}$. The prescribed boundary tractions or Neumann boundary conditions are $\boldsymbol{T}: \partial_{\boldsymbol{T}} \Omega \rightarrow \mathbb{R}^{3}$. Let $\boldsymbol{F}:=\operatorname{Grad} \varphi$ be the deformation gradient. Let also $R \boldsymbol{B}: \Omega \rightarrow \mathbb{R}^{3}$ be the body force, with $R$ the mass density in the reference configuration. Furthermore, introduce the energy functional

$$
\Phi[\boldsymbol{\varphi}]:=\int_{\Omega} A(\boldsymbol{F}, \boldsymbol{Z}) \mathrm{d} V-\int_{\Omega} R \boldsymbol{B} \cdot \boldsymbol{\varphi} \mathrm{d} V-\int_{\partial_{\boldsymbol{T}} \Omega} \boldsymbol{T} \cdot \boldsymbol{\varphi} \mathrm{d} S,
$$

in which $A(\boldsymbol{F}, \boldsymbol{Z})$ is the Helmholtz free-energy density and $\boldsymbol{Z}$ is a collection of internal variables. The weak form of the problem is obtained by minimizing the energy functional $\Phi[\varphi]$ over the Sobolev space $W_{2}^{1}(\Omega)$ that is comprised of all functions that are square-integrable and have square-integrable first derivatives. Define

$$
\mathcal{S}:=\left\{\varphi \in W_{2}^{1}(\Omega): \varphi=\chi \text { on } \partial_{\varphi} \Omega\right\}
$$

and

$$
\mathcal{V}:=\left\{\boldsymbol{\xi} \in W_{2}^{1}(\Omega): \boldsymbol{\xi}=\mathbf{0} \text { on } \partial_{\boldsymbol{\varphi}} \Omega\right\}
$$

where $\boldsymbol{\xi} \in \mathcal{V}$ is a test function. The potential energy is minimized if and only if $\Phi[\varphi] \leq \Phi[\varphi+\epsilon \boldsymbol{\xi}]$ for all $\xi \in \mathcal{V}$ and $\epsilon \in \mathbb{R}$. It is straightforward to show that the minimum of $\Phi[\varphi]$ is the mapping $\varphi \in \mathcal{S}$ that satisfies

$$
D \Phi[\boldsymbol{\varphi}](\boldsymbol{\xi})=\int_{\Omega} \boldsymbol{P}: \operatorname{Grad} \boldsymbol{\xi} \mathrm{d} V-\int_{\Omega} R \boldsymbol{B} \cdot \boldsymbol{\xi} \mathrm{d} V-\int_{\partial_{\boldsymbol{T}} \Omega} \boldsymbol{T} \cdot \boldsymbol{\xi} \mathrm{d} S=0,
$$

where $\boldsymbol{P}=\partial A / \partial \boldsymbol{F}$ denotes the first Piola-Kirchhoff stress. The Euler-Lagrange equation corresponding to the variational statement (4) is

$$
\begin{aligned}
\operatorname{Div} \boldsymbol{P}+R \boldsymbol{B} & =\mathbf{0}, \quad \text { in } \Omega, \\
\boldsymbol{P} \boldsymbol{N} & =\boldsymbol{T}, \quad \text { on } \partial_{\boldsymbol{T}} \Omega, \\
\boldsymbol{\varphi} & =\boldsymbol{\chi}, \quad \text { on } \partial_{\boldsymbol{\varphi}} \Omega .
\end{aligned}
$$

\subsection{Coupling Two or More Subdomains via the Schwarz Alternating Method}

In this section, we describe the Schwarz alternating method for coupling multiple overlapping subdomains. Consider without loss of generality a partition for the domain $\Omega$ into two open subsets or subdomains $\Omega_{1}$ and $\Omega_{2}$ such that $\Omega=\Omega_{1} \cup \Omega_{2}$ and $\Omega_{1} \cap \Omega_{2} \neq \emptyset$ as shown in Figure 1 . 


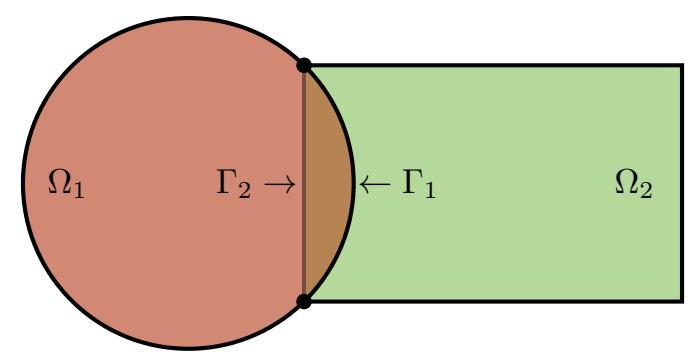

Figure 1: Two subdomains $\Omega_{1}$ and $\Omega_{2}$ and the corresponding boundaries $\Gamma_{1}$ and $\Gamma_{2}$ used by the Schwarz alternating method.

In keeping with other works on the convegence of the Schwarz alternating method, specially as we seek to prove convergence for the finite deformation solid mechanics problem (5), we introduce a set of indices that alternate between the subdomains as

$$
n \in \mathbb{N}^{0}=\{0,1,2, \ldots\}, \quad i=2-n+2\left\lfloor\frac{n}{2}\right\rfloor \in\{1,2\}, \quad j=n+1-2\left\lfloor\frac{n}{2}\right\rfloor \in\{1,2\},
$$

that is $i=1$ and $j=2$ if $n$ is odd, and $i=2$ and $j=1$ if $n$ is even. Introduce the following definitions for each subdomain $i$ :

- Closure: $\bar{\Omega}_{i}:=\Omega_{i} \cup \partial \Omega_{i}$

- Dirichlet boundary: $\partial_{\varphi} \Omega_{i}:=\partial_{\varphi} \Omega \cap \bar{\Omega}_{i}$.

- Neumann boundary: $\partial_{\boldsymbol{T}} \Omega_{i}:=\partial_{\boldsymbol{T}} \Omega \cap \bar{\Omega}_{i}$.

- Schwarz boundary: $\Gamma_{i}:=\partial \Omega_{i} \cap \Omega_{j}$.

Note that with these definitions we guarantee that $\partial_{\boldsymbol{\varphi}} \Omega_{i} \cap \partial_{\boldsymbol{T}} \Omega_{i}=\emptyset, \partial_{\boldsymbol{\varphi}} \Omega_{i} \cap \Gamma_{i}=\emptyset$ and $\partial_{\boldsymbol{T}} \Omega_{i} \cap \Gamma_{i}=\emptyset$. Now define the spaces

$$
\mathcal{S}_{i}:=\left\{\varphi \in W_{2}^{1}\left(\Omega_{i}\right): \varphi=\chi \text { on } \partial_{\varphi} \Omega_{i}, \varphi=P_{\Omega_{j} \rightarrow \Gamma_{i}}\left[\varphi\left(\Omega_{j}\right)\right] \text { on } \Gamma_{i}\right\},
$$

and

$$
\mathcal{V}_{i}:=\left\{\boldsymbol{\xi} \in W_{2}^{1}\left(\Omega_{i}\right): \boldsymbol{\xi}=\mathbf{0} \text { on } \partial_{\boldsymbol{\varphi}} \Omega_{i} \cup \Gamma_{i}\right\},
$$

where the symbol $P_{\Omega_{j} \rightarrow \Gamma_{i}}[\cdot]$ denotes the projection from the subdomain $\Omega_{j}$ onto the Schwarz boundary $\Gamma_{i}$. This projection operator plays a central role in the Schwarz alternating method. Its form and implementation are discussed in subsequent sections. For the moment it is sufficient to assume that the operator is able to project a field $\varphi$ from one subdomain to the Schwarz boundary of the other subdomain.

The Schwarz alternating method solves a sequence of problems on $\Omega_{1}$ and $\Omega_{2}$. The solution $\varphi^{(n)}$ for the $n$-th problem is given by

$$
\boldsymbol{\varphi}^{(n)}= \begin{cases}\operatorname{id}_{\boldsymbol{X}}, & \text { for } n=0 \\ \arg \min _{\boldsymbol{\varphi} \in \mathcal{S}_{i}} \Phi_{i}[\boldsymbol{\varphi}], & \text { for } n>0\end{cases}
$$

where $\operatorname{id}_{\boldsymbol{X}}$ is the identity map that maps $\boldsymbol{X}$ onto itself (i.e. zero displacement), and

$$
\Phi_{i}[\boldsymbol{\varphi}]:=\int_{\Omega_{i}} A(\boldsymbol{F}, \boldsymbol{Z}) \mathrm{d} V-\int_{\Omega_{i}} R \boldsymbol{B} \cdot \boldsymbol{\varphi} \mathrm{d} V-\int_{\partial_{\boldsymbol{T}} \Omega_{i}} \boldsymbol{T} \cdot \boldsymbol{\varphi} \mathrm{d} S .
$$


A better guess, if available, may be used to initialize $\varphi^{(0)}$ on $\Omega_{2}$ rather than the identity map id $\boldsymbol{X}$. The minimization of the functional (10) leads to a variational formulation of the form (4)-(5) for each subdomain as

$$
D \Phi_{i}\left[\boldsymbol{\varphi}^{(n)}\right]\left(\boldsymbol{\xi}^{(i)}\right)=\int_{\Omega_{i}} \boldsymbol{P}: \operatorname{Grad} \boldsymbol{\xi}^{(i)} \mathrm{d} V-\int_{\Omega_{i}} R \boldsymbol{B} \cdot \boldsymbol{\xi}^{(i)} \mathrm{d} V-\int_{\partial_{\boldsymbol{T}} \Omega_{i}} \boldsymbol{T} \cdot \boldsymbol{\xi}^{(i)} \mathrm{d} S=0,
$$

in which $\boldsymbol{\xi}^{(i)} \in \mathcal{V}_{i}$. The corresponding Euler-Lagrange equations are

$$
\begin{aligned}
\operatorname{Div} \boldsymbol{P}+R \boldsymbol{B} & =\mathbf{0}, \quad \text { in } \Omega_{i}, \\
\boldsymbol{P} \boldsymbol{N} & =\boldsymbol{T}, \quad \text { on } \partial_{\boldsymbol{T}} \Omega_{i},
\end{aligned}
$$

with the Dirichlet boundary conditions

$$
\varphi^{(n)}= \begin{cases}\chi, & \text { for } n \geq 0 \text { on } \partial_{\varphi} \Omega_{i} \\ \operatorname{id}_{\boldsymbol{X}}, & \text { for } n=0 \text { on } \Gamma_{i} \\ P_{\Omega_{j} \rightarrow \Gamma_{i}}\left[\varphi^{(n-1)}\left(\Omega_{j}\right)\right], & \text { for } n>0 \text { on } \Gamma_{i} .\end{cases}
$$

Next, on each subdomain $\Omega_{i}$ we introduce the spatial discretization for the field $\varphi^{(n)}$ and its test function $\boldsymbol{\xi}^{(i)}$ as

$$
\begin{aligned}
\boldsymbol{\varphi}_{h}^{(n)}(\boldsymbol{X}) & :=N_{A}^{(i)}(\boldsymbol{X}) \boldsymbol{x}_{A}^{(n)}+N_{a}^{(i)}(\boldsymbol{X}) \boldsymbol{x}_{a}^{(n)}+N_{\alpha}^{(i)}(\boldsymbol{X}) \boldsymbol{x}_{\alpha}^{(n)} \in \mathcal{S}_{i, h}, \\
\boldsymbol{\xi}_{h}^{(i)}(\boldsymbol{X}) & :=N_{B}^{(i)}(\boldsymbol{X}) \boldsymbol{\xi}_{B}^{(i)} \in \mathcal{V}_{i, h},
\end{aligned}
$$

in which $\mathcal{S}_{i, h} \subset \mathcal{S}_{i}$ and $\mathcal{V}_{i, h} \subset \mathcal{V}_{i}$ are finite-dimensional subspaces of $\mathcal{S}_{i}$ and $\mathcal{V}_{i}$, respectively. The formulation allows for different discretizations in $\Omega_{1}$ and $\Omega_{2}$, and therefore also non-matching nodes, different element topologies, or both. Note that we have introduced a partition for the nodal values of the deformed configuration into free, Dirichlet boundary, and Schwarz boundary degrees of freedom, depending on where a node lies as follows:

$$
\boldsymbol{x}_{A}^{(n)} \in \boldsymbol{\varphi}^{(n)}\left(\Omega_{i} \cup \partial_{\boldsymbol{T}} \Omega_{i}\right), \quad \boldsymbol{x}_{a}^{(n)} \in \boldsymbol{\varphi}^{(n)}\left(\partial_{\boldsymbol{\varphi}} \Omega_{i}\right), \quad \boldsymbol{x}_{\alpha}^{(n)} \in \boldsymbol{\varphi}^{(n)}\left(\Gamma_{i}\right) .
$$

We denote free degrees of freedom with uppercase Latin indices $(A, B)$, Dirichlet boundary degrees of freedom with lowercase Latin indices $(a, b)$, and Schwarz boundary degrees of freedom with lowercase Greek indices $(\alpha, \beta)$. Thus, for each of the subdomains, $N_{A}^{(i)}$ and $N_{B}^{(i)}$ are the same basis functions corresponding to the free degrees of freedom, $N_{a}^{(i)}$ are basis functions corresponding to the Dirichlet boundary degrees of freedom, and $N_{\alpha}^{(i)}$ are basis functions corresponding to the Schwarz boundary degrees of freedom. In addition, $\boldsymbol{\xi}_{B}^{(i)}$ are nodal values for the test function, and $\boldsymbol{\xi}_{b}^{(i)}=\boldsymbol{\xi}_{\beta}^{(i)}=\mathbf{0}$ by virtue of the homogeneous condition for the test functions (8).

Introducing these discretizations into the variational statements (11) gives

$$
D \Phi_{i}\left[\boldsymbol{\varphi}_{h}^{(n)}\right]\left(\boldsymbol{\xi}_{h}^{(i)}\right)=\boldsymbol{R}_{A}^{(n)}\left[\boldsymbol{\varphi}_{h}^{(n)}\right] \cdot \boldsymbol{\xi}_{A}^{(i)}=0,
$$

together with the discrete statements of equilibrium

$$
\boldsymbol{R}_{A}^{(n)}\left[\boldsymbol{\varphi}_{h}^{(n)}\right]:=\int_{\Omega_{i}} \boldsymbol{P}: \boldsymbol{B}_{A}^{(i)} \mathrm{d} V-\int_{\Omega_{i}} R \boldsymbol{B} N_{A}^{(i)} \mathrm{d} V-\int_{\partial_{\boldsymbol{T}} \Omega_{i}} \boldsymbol{T} N_{A}^{(i)} \mathrm{d} S=\mathbf{0},
$$

in which $\mathcal{B}_{A}^{(i)}$ is a third-order tensor defined as

$$
\boldsymbol{\mathcal { B }}_{A}^{(i)}(\boldsymbol{X}):=\delta_{p r} \frac{\partial N_{A}^{(i)}(\boldsymbol{X})}{\partial X_{Q}} \boldsymbol{e}_{p} \otimes \boldsymbol{E}_{Q} \otimes \boldsymbol{e}_{r},
$$


where $\boldsymbol{E}_{Q}$ and $\boldsymbol{e}_{p}$ are orthonormal bases for the reference and current configurations, respectively. This tensor plays the role of the gradient operator such that

$$
\boldsymbol{F}^{(n)}(\boldsymbol{X})=\mathcal{B}_{A}^{(i)}(\boldsymbol{X}) \boldsymbol{x}_{A}^{(n)} \quad \text { on } \quad \Omega_{i} .
$$

In summary, the Schwarz alternating method is described in Algorithm 1. This procedure may be extended in a straightforward way to more than two subdomains.

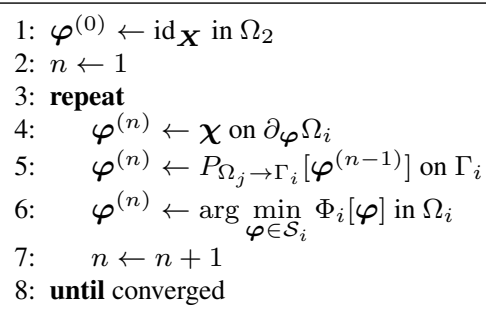

$\triangleright$ initialize to zero displacement or a better guess in $\Omega_{2}$

Algorithm 1: Schwarz Alternating Method

We emphasize that, although we have only considered the specific case of the finite element method, the Schwarz alternating method described in this work can be used to couple subdomains for which the solution is found by other methods, not necessarily all the same. Moreover, within the context of finite elements, different interpolation schemes and element topologies may be used in different subdomains.

\subsection{Linearization}

To obtain solutions for the nonlinear discrete variational statement (17), one may apply Newton-type methods or other related linearization-based techniques such that incremental solutions may be obtained at each load step. In this setting, the Schwarz alternating method of Algorithm 1 is performed at each load step. Then the initial solution $\varphi^{(0)}$ is set to the converged solution of the previous step. Let us now introduce the incremental indices $m$ and $m+1$ with the understanding that further developments take place at time $t \in\left[t_{m}, t_{m+1}\right]$, where $m \in \mathbb{N}^{0}=\{0,1,2, \ldots\} .{ }^{1}$ The solution $\varphi_{h}^{(n)}$, is known at time $t_{m}$ and we wish to advance it to time $t_{m+1}$. Assuming that all loads are conservative, the linear approximation of the statement of equilibrium for $\varphi_{h}^{(n)}$ is

$$
\mathbf{0}=\boldsymbol{R}_{A}^{(n)}\left[\boldsymbol{\varphi}_{h}^{(n, m+1)}\right] \approx \boldsymbol{R}_{A}^{(n)}\left[\boldsymbol{\varphi}_{h}^{(n, m)}\right]+D \boldsymbol{R}_{A}^{(n)}\left[\boldsymbol{\varphi}_{h}^{(n, m)}\right]\left(\triangle \boldsymbol{\varphi}_{h}^{(n)}\right),
$$

where $\triangle \boldsymbol{\varphi}_{h}^{(n)}:=\boldsymbol{\varphi}_{h}^{(n, m+1)}-\boldsymbol{\varphi}_{h}^{(n, m)}$ denotes the linear increment from $\boldsymbol{\varphi}_{h}^{(n, m)}$ to $\varphi_{h}^{(n, m+1)}$ for the $(m+1)$ th step. Introducing the discretization (14) for the increments and after some straightforward algebraic manipulation, the directional derivative with respect to $\varphi_{h}^{(n, m)}$ yields

$$
D \boldsymbol{R}_{A}^{(n)}\left[\boldsymbol{\varphi}_{h}^{(n, m)}\right]\left(\triangle \boldsymbol{\varphi}_{h}^{(n)}\right)=\boldsymbol{K}_{A B}^{(n)} \cdot \triangle \boldsymbol{x}_{B}^{(n)}+\boldsymbol{K}_{A b}^{(n)} \cdot \triangle \boldsymbol{x}_{b}^{(n)}+\boldsymbol{K}_{A \beta}^{(n)} \cdot \triangle \boldsymbol{x}_{\beta}^{(n)}
$$

where

$$
\triangle \boldsymbol{x}_{B}^{(n)}:=\boldsymbol{x}_{B}^{(n, m+1)}-\boldsymbol{x}_{B}^{(n, m)}, \quad \triangle \boldsymbol{x}_{b}^{(n)}:=\boldsymbol{x}_{b}^{(n, m+1)}-\boldsymbol{x}_{b}^{(n, m)}, \quad \triangle \boldsymbol{x}_{\beta}^{(n)}:=\boldsymbol{x}_{\beta}^{(n, m+1)}-\boldsymbol{x}_{\beta}^{(n, m)},
$$

\footnotetext{
${ }^{1}$ This time $t$ plays the role of a continuation variable for the quasi-static problem addressed here. It does not imply a dynamic problem.
} 
and

$$
\begin{aligned}
\boldsymbol{K}_{A B}^{(n)} & :=\int_{\Omega_{i}} \mathcal{B}_{A}^{(i)^{\mathrm{T}}}: \mathbb{C}^{(n)}: \mathcal{B}_{B}^{(i)} \mathrm{d} V, \\
\boldsymbol{K}_{A b}^{(n)} & :=\int_{\Omega_{i}} \mathcal{B}_{A}^{(i)^{\mathrm{T}}}: \mathbb{C}^{(n)}: \mathcal{B}_{b}^{(i)} \mathrm{d} V, \\
\boldsymbol{K}_{A \beta}^{(n)} & :=\int_{\Omega_{i}} \mathcal{B}_{A}^{(i)^{\mathrm{T}}}: \mathbb{C}^{(n)}: \mathcal{B}_{\beta}^{(i)} \mathrm{d} V,
\end{aligned}
$$

in which $\mathcal{B}_{a}^{(i)}$ and $\mathcal{B}_{\alpha}^{(i)}$ are defined in similar fashion as $\mathcal{B}_{A}^{(i)}$ from (18). Additionally

$$
\mathbb{C}^{(n)}\left(\boldsymbol{F}^{(n)}\right):=\frac{\partial^{2} A}{\partial \boldsymbol{F} \partial \boldsymbol{F}}\left(\boldsymbol{F}^{(n)}\right) .
$$

For simplicity in notation and unless otherwise stated, henceforth we omit the incremental indices $m$ and $m+1$. Now define a partition for the projection $P_{\Omega_{j} \rightarrow \Gamma_{i}}[\cdot]$ introduced in (7) as

$$
P_{\Omega_{j} \rightarrow \Gamma_{i}}[\cdot]=\left\{\begin{array}{c}
P_{\Omega_{j} \cup \partial_{T} \Omega_{j} \rightarrow \Gamma_{i}}[\cdot] \\
P_{\partial_{\varphi} \Omega_{j} \rightarrow \Gamma_{i}}[\cdot] \\
P_{\Gamma_{j} \rightarrow \Gamma_{i}}[\cdot]
\end{array}\right\}
$$

and let

$$
\begin{aligned}
\boldsymbol{P}_{i j} & :=P_{\Omega_{j} \cup \partial_{\boldsymbol{T}} \Omega_{j} \rightarrow \Gamma_{i}}[\cdot] \\
\boldsymbol{Q}_{i j} & :=P_{\partial_{\boldsymbol{\varphi}} \Omega_{j} \rightarrow \Gamma_{i}}[\cdot] \\
\boldsymbol{G}_{i j} & :=P_{\Gamma_{j} \rightarrow \Gamma_{i}}[\cdot]
\end{aligned}
$$

where $\boldsymbol{P}_{i j}, \boldsymbol{Q}_{i j}$, and $\boldsymbol{G}_{i j}$ are the projection matrices from the free, Dirichlet boundary, and Schwarz boundary degrees of freedom of subdomain $j$ to the Schwarz boundary degrees of freedom of subdomain $i$, respectively. This results in the explicit representation of the Schwarz boundary degrees of freedom of one subdomain in terms of the degrees of freedom of the other subdomain as

$$
\boldsymbol{x}_{\beta}^{(n)}=\left[\begin{array}{lll}
\boldsymbol{P}_{i j} & \boldsymbol{Q}_{i j} & \boldsymbol{G}_{i j}
\end{array}\right]\left\{\begin{array}{l}
\boldsymbol{x}_{B}^{(n-1)} \\
\boldsymbol{x}_{b}^{(n-1)} \\
\boldsymbol{x}_{\beta}^{(n-1)}
\end{array}\right\} .
$$

Introducing the Dirichlet boundary conditions (13) for the degrees of freedom on the Dirichlet boundary $\partial_{\varphi} \Omega_{i}$ gives

$$
\boldsymbol{x}_{b}^{(n)}=\chi_{b}^{(i)} \Rightarrow \triangle \boldsymbol{x}_{b}^{(n)}=\mathbf{0} .
$$

Let us consider now the degrees of freedom that reside at the Schwarz boundaries $\Gamma_{i}$. They may be treated in two different ways, which lead to different variants of the Schwarz alternating method, as shown next.

\section{$\underline{\text { Schwarz Boundaries as Dirichlet Boundaries }}$}

In the original Schwarz method, the degrees of freedom on the Schwarz boundaries $\Gamma_{i}$ are treated in the same manner as the degrees of freedom on the Dirichlet boundaries $\partial_{\varphi} \Omega_{i}$ : they are all subject to Dirichlet 
boundary conditions $[55,58,41,2]$. For each Schwarz iteration this results in

$$
\boldsymbol{x}_{\beta}^{(n)}=\left[\begin{array}{lll}
\boldsymbol{P}_{i j} & \boldsymbol{Q}_{i j} & \boldsymbol{G}_{i j}
\end{array}\right]\left\{\begin{array}{l}
\boldsymbol{x}_{B}^{(n-1)} \\
\boldsymbol{x}_{b}^{(n-1)} \\
\boldsymbol{x}_{\beta}^{(n-1)}
\end{array}\right\}, \quad \triangle \boldsymbol{x}_{\beta}^{(n)}=\mathbf{0}
$$

Introducing $\triangle \boldsymbol{x}_{b}^{(n)}=\mathbf{0}$ from (28) and $\triangle \boldsymbol{x}_{\beta}^{(n)}=\mathbf{0}$ from (29) into the approximation (20) and linearization (21) for the residual, and recalling the we dropped the indices $m$ and $m+1$, leads to

$$
\boldsymbol{K}_{A B}^{(n)} \triangle \boldsymbol{x}_{B}^{(n)}=-\boldsymbol{R}_{A}^{(n)}
$$

This may be represented explicitly for the two subdomains in block form for each Schwarz iterarion. Hence, dropping the index $n$ and using 1 and 2 for each subdomain instead (with the understanding that the context is still the Schwarz iteration), results in

$$
\left(\begin{array}{cc}
\boldsymbol{K}_{A B}^{(1)} & \mathbf{0} \\
\mathbf{0} & \boldsymbol{K}_{A B}^{(2)}
\end{array}\right)\left\{\begin{array}{l}
\Delta \boldsymbol{x}_{B}^{(1)} \\
\triangle \boldsymbol{x}_{B}^{(2)}
\end{array}\right\}=\left\{\begin{array}{c}
-\boldsymbol{R}_{A}^{(1)} \\
-\boldsymbol{R}_{A}^{(2)}
\end{array}\right\}
$$

Note that the block linear system (31) possesses the following desirable properties:

1. It is a block-diagonal system that may be solved independently for each block with a conventional linear solver or simultaneously with a block solver.

2. The coupling between the two subdomains takes place through the right hand side only, which significantly decreases the complexity of implementation.

3. If the diagonal blocks are well-conditioned, the block matrix is also well-conditioned. This occurs, for example, when the subdomains are discretized with meshes that have significantly different levels of refinement. This is not necessarily the case in a system with off-diagonal blocks.

\section{Elimination of the Schwarz Boundaries Degrees of Freedom}

In the domain decomposition community it is common to altogether eliminate the degrees of freedom on the Schwarz boundaries $\Gamma_{i}$ by explicitly introducing the projection (27) into the linearization of the residual (21) $[57,64,40,50]$. Using $\Delta \boldsymbol{x}_{b}^{(n)}=\mathbf{0}$ from (28) in the projection (27) gives

$$
\boldsymbol{x}_{\beta}^{(n)}=\left[\begin{array}{lll}
\boldsymbol{P}_{i j} & \boldsymbol{Q}_{i j} & \boldsymbol{G}_{i j}
\end{array}\right]\left\{\begin{array}{l}
\boldsymbol{x}_{B}^{(n-1)} \\
\boldsymbol{x}_{b-1)}^{(n-1)} \\
\boldsymbol{x}_{\beta}^{(n-1)}
\end{array}\right\} \Rightarrow \triangle \boldsymbol{x}_{\beta}^{(n)}=\left[\begin{array}{ll}
\boldsymbol{P}_{i j} & \boldsymbol{G}_{i j}
\end{array}\right]\left\{\begin{array}{l}
\triangle \boldsymbol{x}_{B}^{(n-1)} \\
\triangle \boldsymbol{x}_{\beta}^{(n-1)}
\end{array}\right\} .
$$

Introducing this expression for $\Delta \boldsymbol{x}_{\beta}^{(n)}$ and again $\Delta \boldsymbol{x}_{b}^{(n)}=\mathbf{0}$ from (28) into the approximation (20) and linearization (21) for the residual, and recalling the we dropped the indices $m$ and $m+1$, results in

$$
\boldsymbol{K}_{A B}^{(n)} \triangle \boldsymbol{x}_{B}^{(n)}+\boldsymbol{K}_{A \beta}^{(n)} \triangle \boldsymbol{x}_{\beta}^{(n)}=-\boldsymbol{R}_{A}^{(n)}
$$

This system may also be represented explicitly for the two subdomains in block form for each Schwarz iterarion. Applying the reduced projection for $\triangle \boldsymbol{x}_{\beta}^{(n)}$ in (32) and after some algebraic manipulation (dropping 
the index $n$ and using 1 and 2 for each subdomain instead) results in the coupled system

$$
\left(\begin{array}{cc}
\boldsymbol{K}_{A B}^{(1)}+\boldsymbol{K}_{A \beta}^{(1)} \boldsymbol{H}_{11} & \boldsymbol{K}_{A \beta}^{(1)} \boldsymbol{H}_{12} \\
\boldsymbol{K}_{A \beta}^{(2)} \boldsymbol{H}_{21} & \boldsymbol{K}_{A B}^{(2)}+\boldsymbol{K}_{A \beta}^{(2)} \boldsymbol{H}_{22}
\end{array}\right)\left\{\begin{array}{c}
\triangle \boldsymbol{x}_{B}^{(1)} \\
\triangle \boldsymbol{x}_{B}^{(2)}
\end{array}\right\}=\left\{\begin{array}{c}
-\boldsymbol{R}_{A}^{(1)} \\
-\boldsymbol{R}_{A}^{(2)}
\end{array}\right\}
$$

where $\boldsymbol{H}_{i i}:=\left(\boldsymbol{I}_{i}-\boldsymbol{G}_{i j} \boldsymbol{G}_{j i}\right)^{-1} \boldsymbol{G}_{i j} \boldsymbol{P}_{j i}$ and $\boldsymbol{H}_{i j}:=\left(\boldsymbol{I}_{i}-\boldsymbol{G}_{i j} \boldsymbol{G}_{j i}\right)^{-1} \boldsymbol{P}_{i j}$ (no summation), and in which $\boldsymbol{I}_{i}$ is the identity matrix corresponding to the number of degrees of freedom for the Schwarz boundary $\Gamma_{i}$. Note that this linear system is analogous to the systems obtained for the Schwarz alternating method as used in domain decomposition for partial differential equations (cf. [57, 64, 40, 50]). Also note that assuming that $\boldsymbol{P}_{12}=\mathbf{0}$ and $\boldsymbol{P}_{21}=\mathbf{0}$, the block-non-diagonal system (34) reduces to the block-diagonal system (31). The implementation of the block-non-diagonal system (34) may prove difficult and cumbersome, unless the code in which it is to be introduced is already amenable to such matrix forms.

\subsection{Variants of the Schwarz Alternating Method}

Next, we propose four variants of the Schwarz alternating method, described below, that can be derived from the block-diagonal system (31) or the block-non-diagonal system (34).

\section{Full Schwarz (Algorithm 2)}

This is a direct application of the method as devised originally by Schwarz [55]. The solution of the coupled nonlinear systems (17) is obtained by applying the alternating iterative procedure described in Algorithm 1. The solution for each subdomain is obtained by using the standard Newton method with the Dirichlet (28) and Schwarz (29) boundary conditions. The linear system for each subdomain is the corresponding row of the block-diagonal system (31). For a computer implementation, an outer loop that controls the Schwarz iterations and the projection of the solution onto the Schwarz boundaries (27) need to be wrapped around the solver for the nonlinear system of each subdomain. Both the Newton and Schwarz loops use a tight tolerance, for example, machine precision $\left(\epsilon_{\text {machine }}\right)$.

\section{Modified Schwarz (Algorithm 3)}

Within the context of a Newton solution scheme, the block-diagonal system (31) may be intepreted as the Hessian of the coupled system, and thus may be used to solve for the solution of both subdomains at the same time using a block solver or separately by a conventional solver. Furthermore, the projection (27) is effected in an alternating fashion for each Newton iteration. This strongly motivates the notion of combining the Newton and Schwarz iterations for each increment $m$ into a single procedure by using the the block-diagonal system (31) as the Hessian for the Newton iteration, using a tight convergence tolerance. ${ }^{2}$ This version is particularly attractive from the point of view of computer implementation, as it is the least intrusive of all the four variants presented here for two reasons: (1) it completely by-passes the Schwarz iteration and thus there is no need for the Schwarz loop, and (2) a conventional solver may be used for each subdomain, without the need for a block solver.

\footnotetext{
${ }^{2}$ The notion of using a single iterative scheme to combine both the nonlinear solution method and the coupling method for a block system was explored by Cervera, Codina, and Galindo [15]. The authors show that even for a weakly nonlinear system the iterations due to the nonlinear solution method govern the overall process, therefore the coupling is achieved automatically and inexpensively as a part of the Newton method. Note that the authors did not address specifically the Schwarz alternating method, as we consider here.
} 


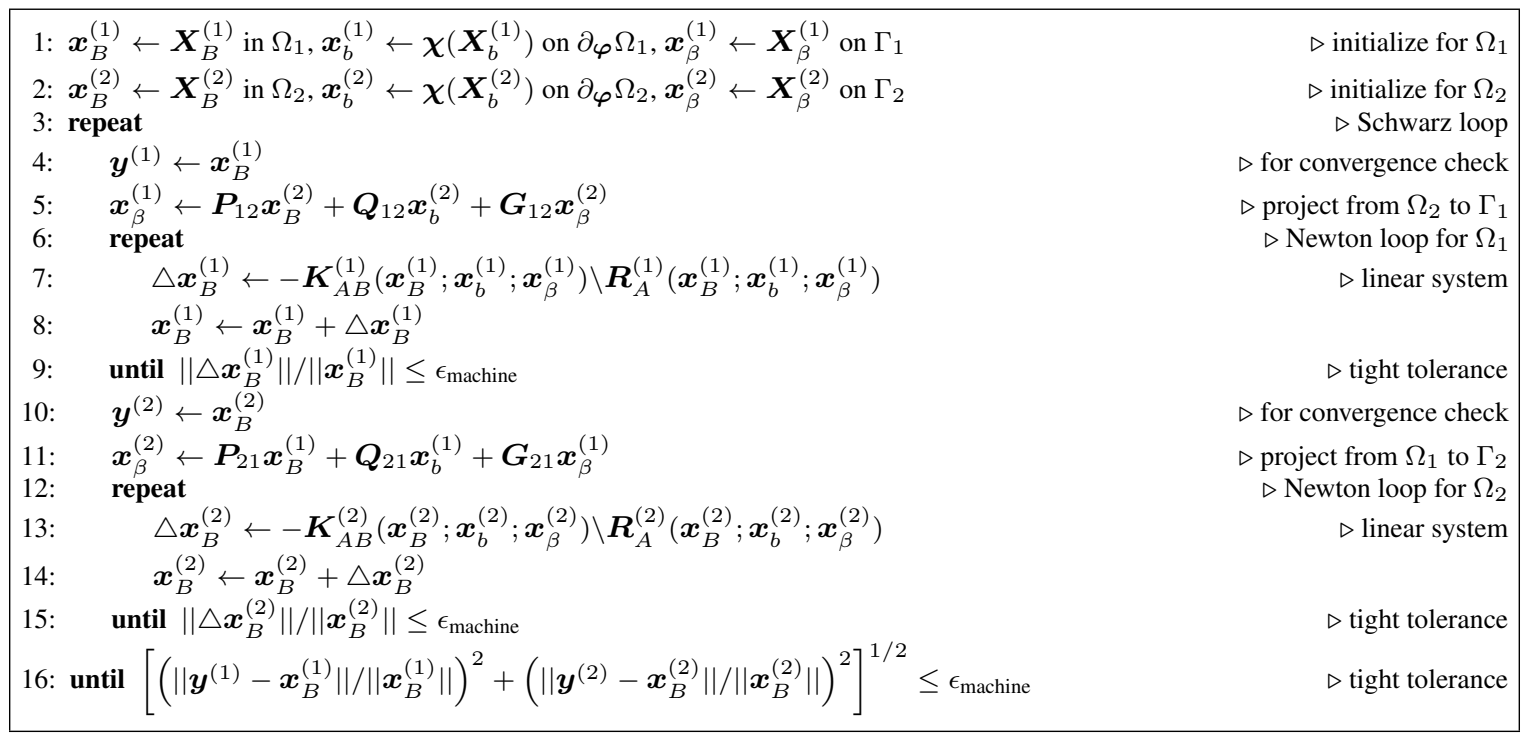

Algorithm 2: Full Schwarz Method

\begin{tabular}{|llr|}
\hline 1: $\boldsymbol{x}_{B}^{(1)} \leftarrow \boldsymbol{X}_{B}^{(1)}$ in $\Omega_{1}, \boldsymbol{x}_{b}^{(1)} \leftarrow \boldsymbol{\chi}\left(\boldsymbol{X}_{b}^{(1)}\right)$ on $\partial_{\boldsymbol{\varphi}} \Omega_{1}, \boldsymbol{x}_{\beta}^{(1)} \leftarrow \boldsymbol{X}_{\beta}^{(1)}$ on $\Gamma_{1}$ & $\triangleright$ initialize for $\Omega_{1}$ \\
2: $\boldsymbol{x}_{B}^{(2)} \leftarrow \boldsymbol{X}_{B}^{(2)}$ in $\Omega_{2}, \boldsymbol{x}_{b}^{(2)} \leftarrow \boldsymbol{\chi}\left(\boldsymbol{X}_{b}^{(2)}\right)$ on $\partial_{\boldsymbol{\varphi}} \Omega_{2}, \boldsymbol{x}_{\beta}^{(2)} \leftarrow \boldsymbol{X}_{\beta}^{(2)}$ on $\Gamma_{2}$ & $\triangleright$ initialize for $\Omega_{2}$ \\
3: repeat & Newton-Schwarz loop \\
4: $\quad \boldsymbol{x}_{\beta}^{(1)} \leftarrow \boldsymbol{P}_{12} \boldsymbol{x}_{B}^{(2)}+\boldsymbol{Q}_{12} \boldsymbol{x}_{b}^{(2)}+\boldsymbol{G}_{12} \boldsymbol{x}_{\beta}^{(2)}$ & $\triangleright$ project from $\Omega_{2}$ to $\Gamma_{1}$ \\
5: $\quad \triangle \boldsymbol{x}_{B}^{(1)} \leftarrow-\boldsymbol{K}_{A B}^{(1)}\left(\boldsymbol{x}_{B}^{(1)} ; \boldsymbol{x}_{b}^{(1)} ; \boldsymbol{x}_{\beta}^{(1)}\right) \backslash \boldsymbol{R}_{A}^{(1)}\left(\boldsymbol{x}_{B}^{(1)} ; \boldsymbol{x}_{b}^{(1)} ; \boldsymbol{x}_{\beta}^{(1)}\right)$ & $\triangleright$ linear system \\
6: $\quad \boldsymbol{x}_{B}^{(1)} \leftarrow \boldsymbol{x}_{B}^{(1)}+\triangle \boldsymbol{x}_{B}^{(1)}$ & $\triangleright$ project from $\Omega_{1}$ to $\Gamma_{2}$ \\
7: $\quad \boldsymbol{x}_{\beta}^{(2)} \leftarrow \boldsymbol{P}_{21} \boldsymbol{x}_{B}^{(1)}+\boldsymbol{Q}_{21} \boldsymbol{x}_{b}^{(1)}+\boldsymbol{G}_{21} \boldsymbol{x}_{\beta}^{(1)}$ & $\triangleright$ linear system \\
8: $\quad \triangle \boldsymbol{x}_{B}^{(2)} \leftarrow-\boldsymbol{K}_{A B}^{(2)}\left(\boldsymbol{x}_{B}^{(2)} ; \boldsymbol{x}_{b}^{(2)} ; \boldsymbol{x}_{\beta}^{(2)}\right) \backslash \boldsymbol{R}_{A}^{(2)}\left(\boldsymbol{x}_{B}^{(2)} ; \boldsymbol{x}_{b}^{(2)} ; \boldsymbol{x}_{\beta}^{(2)}\right)$ & \\
9: $\quad \boldsymbol{x}_{B}^{(2)} \leftarrow \boldsymbol{x}_{B}^{(2)}+\triangle \boldsymbol{x}_{B}^{(2)}$ & \\
10: until $\left[\left(\left\|\triangle \boldsymbol{x}_{B}^{(1)}\right\| /\left\|\boldsymbol{x}_{B}^{(1)}\right\|\right)^{2}+\left(\left\|\triangle \boldsymbol{x}_{B}^{(2)}\right\| /\left\|\boldsymbol{x}_{B}^{(2)}\right\|\right)^{2}\right]^{1 / 2} \leq \epsilon_{\text {machine }}$ & \\
&
\end{tabular}

Algorithm 3: Modified Schwarz Method 


\section{Inexact Schwarz (Algorithm 4)}

In the Full Schwarz variant described above, the Schwarz and Newton loops use a tight convergence tolerance $\epsilon_{\text {machine }}$. It seems appealing to modify this algorithm in the spirit of Inexact Newton methods [44] by loosening the convergence tolerance for the Newton loop. We refer to this variant of the Full Schwarz algorithm as "Inexact Schwarz". This is essentially the Full Schwarz variant for which the solution for each subdomain is loosely approximated by a Newton scheme by setting the corresponding convergence tolerance to a relatively loose value. It will be shown that the Inexact Schwarz method behaves as an intermediate between the Full Schwarz and the Modified Schwarz variants described above.

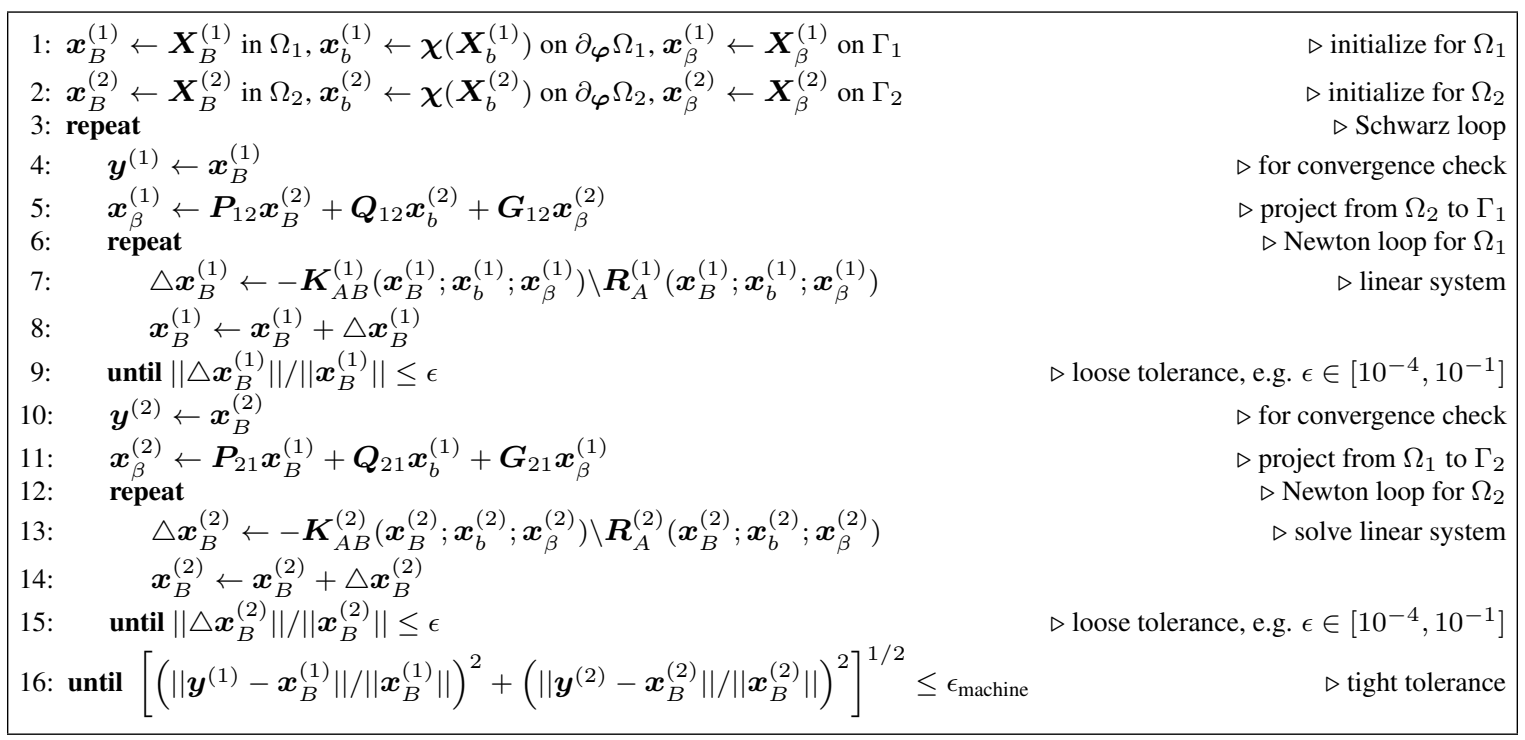

Algorithm 4: Inexact Schwarz Method

\section{Monolithic Schwarz (Algorithm 5)}

This is the same as the Modified Schwarz variant, except that instead of using the block-diagonal system (31) as the Hessian for the Newton method, it uses the block-non-diagonal system (34) instead. A tight Newton-Schwarz tolerance is used. Since this system has off-diagonal blocks, it needs to be solved by means of a block solver (hence the name Monolithic), whereas for the Modified Schwarz variant there is the option of solving for each subdomain independently.

\section{Convergence of the Schwarz Alternating Method}

In this section, we provide some conditions under which the Full Schwarz method described in Section 2.2 converges. We present the proof of convergence for two subdomains in Appendix A. The proof combines notions and concepts from several authors, namely Sobolev [58], Lions [38], Badea [4], Scarpini [54], and Gürses [29]. We emphasize that although Theorem 1 proves convergence for the specific case of only two subdomains, it can be extended to the more general case of an arbitrary number of overlapping subdomains 


\begin{tabular}{llr|}
\hline 1: & $\boldsymbol{x}_{B}^{(1)} \leftarrow \boldsymbol{X}_{B}^{(1)}$ in $\Omega_{1}, \boldsymbol{x}_{b}^{(1)} \leftarrow \boldsymbol{\chi}\left(\boldsymbol{X}_{b}^{(1)}\right)$ on $\partial_{\boldsymbol{\varphi}} \Omega_{1}$, & \\
2: & $\boldsymbol{x}_{B}^{(2)} \leftarrow \boldsymbol{X}_{B}^{(2)}$ in $\Omega_{2}, \boldsymbol{x}_{b}^{(2)} \leftarrow \boldsymbol{\chi}\left(\boldsymbol{X}_{b}^{(2)}\right)$ on $\partial_{\boldsymbol{\varphi}} \Omega_{2}$, & initialize for $\Omega_{1}$ \\
3: & repeat & initialize for $\Omega_{2}$ \\
4: & $\left\{\begin{array}{l}\triangle \boldsymbol{x}_{B}^{(1)} \\
\triangle \boldsymbol{x}_{B}^{(2)}\end{array}\right\} \leftarrow\left(\begin{array}{cc}\boldsymbol{K}_{A B}^{(1)}+\boldsymbol{K}_{A \beta}^{(1)} \boldsymbol{H}_{11} & \boldsymbol{K}_{A \beta}^{(1)} \boldsymbol{H}_{12} \\
\boldsymbol{K}_{A \beta}^{(2)} \boldsymbol{H}_{21} & \boldsymbol{K}_{A B}^{(2)}+\boldsymbol{K}_{A \beta}^{(2)} \boldsymbol{H}_{22}\end{array}\right) \backslash\left\{\begin{array}{c}-\boldsymbol{R}_{A}^{(1)} \\
-\boldsymbol{R}_{A}^{(2)}\end{array}\right\}$ & $\triangleright$ linear system \\
5: $\quad \boldsymbol{x}_{B}^{(1)} \leftarrow \boldsymbol{x}_{B}^{(1)}+\triangle \boldsymbol{x}_{B}^{(1)}$ & \\
6: $\quad \boldsymbol{x}_{B}^{(2)} \leftarrow \boldsymbol{x}_{B}^{(2)}+\triangle \boldsymbol{x}_{B}^{(2)}$ & \\
7: & until $\left[\left(\left\|\triangle \boldsymbol{x}_{B}^{(1)}\right\| /\left\|\boldsymbol{x}_{B}^{(1)}\right\|\right)^{2}+\left(\left\|\triangle \boldsymbol{x}_{B}^{(2)}\right\| /\left\|\boldsymbol{x}_{B}^{(2)}\right\|\right)^{2}\right]^{1 / 2} \leq \epsilon_{\text {machine }}$ & $\triangleright$ tight tolerance \\
\hline
\end{tabular}

Algorithm 5: Monolithic Schwarz Method

$[38,37,4]$. Although we do not provide here formal convergence proofs for the remaining variants of the Schwarz method, we offer some numerical results illustrating their convergence in Section 4.

Consider the energy functional $\Phi[\varphi]$ defined in (1). We will denote by $(\cdot, \cdot)$ the usual $L^{2}$ inner product over $\Omega$, that is,

$$
\left(\psi_{1}, \psi_{2}\right):=\int_{\Omega} \psi_{1} \cdot \psi_{2} \mathrm{~d} V
$$

for $\boldsymbol{\psi}_{1}, \boldsymbol{\psi}_{2} \in W_{2}^{1}(\Omega)$, with corresponding norm $\|\cdot\|$. The proof of the convergence of the Schwarz alternating method requires that the functional $\Phi[\varphi]$ satisfy the following properties over the space $\mathcal{S}$ defined in (2):

1. $\Phi[\varphi]$ is coercive.

2. $\Phi[\varphi]$ is Fréchet differentiable, with $\Phi^{\prime}[\varphi]$ denoting its Fréchet derivative.

3. $\Phi[\varphi]$ is strictly convex.

4. $\Phi[\varphi]$ is lower semi-continuous.

5. $\Phi^{\prime}[\varphi]$ is uniformly continuous on $\mathcal{K}_{R}$, where

$$
\mathcal{K}_{R}:=\{\varphi \in \mathcal{S}: \Phi[\varphi]<R, R \in \mathbb{R}, R<\infty\} .
$$

It can be shown that the energy functional $\Phi[\varphi]$ defined in (1) is strictly convex in $\mathcal{S}$ (property 3 ) provided that the Helmholtz free-energy density $A(\boldsymbol{F}, \boldsymbol{Z})$ is a quasi-convex function of $\boldsymbol{F}$ [29]. Properties 1, 2, 4 and 5 follow from the strict convexity of $\Phi[\varphi]$. Next, define two additional sets of spaces

$$
\tilde{\mathcal{S}}_{n}:=\left\{\boldsymbol{\varphi} \in \mathcal{S}: \varphi=P_{\Omega_{j} \rightarrow \Gamma_{i}}\left[\boldsymbol{\varphi}^{(n-1)}\left(\Omega_{j}\right)\right] \text { on } \Gamma_{i}, \boldsymbol{\varphi}=\boldsymbol{\varphi}^{(n-1)} \text { on } \Omega \backslash \Omega_{i}\right\},
$$

and

$$
\tilde{\mathcal{V}}_{i}:=\left\{\boldsymbol{\xi} \in \mathcal{S}: \boldsymbol{\xi}=\mathbf{0} \text { in } \Omega \backslash \Omega_{i}\right\},
$$

where $i=1$ and $j=2$ if $n$ is odd, and $i=2$ and $j=1$ if $n$ is even for $n \in\{1,2, \ldots\}$ as given by (6) and with the function $\varphi^{(0)} \in \mathcal{S}$ an initial guess. Note that the spaces $\tilde{\mathcal{S}}_{n}$ in (37) are extensions of the spaces $\mathcal{S}_{i}$ in (7) to the entire domain $\Omega$. With this notation in place, the solution to the $n$-th problem (9)-(13) can be recast as

$$
\tilde{\boldsymbol{\varphi}}^{(n)}= \begin{cases}\operatorname{id}_{\boldsymbol{X}}, & \text { for } n=0 ; \\ \arg \min _{\boldsymbol{\varphi} \in \tilde{\mathcal{S}}_{n}} \Phi[\boldsymbol{\varphi}], & \text { for } n>0 .\end{cases}
$$


Remark that [54]

$$
\tilde{\mathcal{S}}_{n}=\tilde{\varphi}^{(n-1)}+\tilde{\mathcal{V}}_{i} \quad \text { for } \quad \tilde{\varphi}^{(n-1)} \in \tilde{\mathcal{S}}_{n-1} \Rightarrow \tilde{\varphi}^{(n-1)} \in \tilde{\mathcal{S}}_{n}
$$

Theorem 1. Assume that the energy functional $\Phi[\varphi]$ satisfies properties $1-5$ above. Consider the Schwarz alternating method of Section 2 defined by (9)-(13) and its equivalent form (39). Then

(a) $\Phi\left[\tilde{\boldsymbol{\varphi}}^{(0)}\right] \geq \Phi\left[\tilde{\boldsymbol{\varphi}}^{(1)}\right] \geq \cdots \geq \Phi\left[\tilde{\boldsymbol{\varphi}}^{(n-1)}\right] \geq \Phi\left[\tilde{\boldsymbol{\varphi}}^{(n)}\right] \geq \cdots \geq \Phi[\boldsymbol{\varphi}]$, where $\boldsymbol{\varphi}$ is the minimizer of $\Phi[\boldsymbol{\varphi}]$ over $\mathcal{S}$.

(b) the sequence $\left\{\tilde{\varphi}^{(n)}\right\}$ defined in (39) converges to the minimizer $\varphi$ of $\Phi[\varphi]$ in $\mathcal{S}$.

(c) the Schwarz minimum values $\Phi\left[\tilde{\varphi}^{(n)}\right]$ converge monotonically to the minimum value $\Phi[\varphi]$ in $\mathcal{S}$ starting from any initial guess $\tilde{\varphi}^{(0)}$.

(d) if $\Phi^{\prime}[\varphi]$ is Lipschitz continuous in a neighborhood of $\varphi$, then the sequence $\left\{\tilde{\varphi}^{(n)}\right\}$ converges geometrically to the minimizer $\varphi .^{3}$

Proof. See Appendix A.

Finally, while most of works cited above present their analysis for the specific case of two subdomains, extension to multiple subdomains is in general straightforward. The case of multiple subdomains is considered specifically in Lions [38], Badea [4], and Li-Shan and Evans [37].

\section{Numerical Examples}

In this section, we present numerical examples of the behavior of the Schwarz alternating method for two different implementations. First, we briefly describe the two implementations, one in MATLAB and the other in the open-source ALBANY finite element code [35]. Next, we discuss the error measures used throughout the numerical examples. Then, we continue with four examples that demonstrate different features of the Schwarz alternating method and our implementations. The first example, a one-dimensional singular bar, is used to demonstrate the behavior of the four Schwarz variants of Section 2.4. The second example, a cuboid body of square base, aims to study the effect of the size of the overlap region on the convergence of the method. The objective of the third example, a notched cylinder, is to analyze the numerical error in the results and the demonstrate the ability of the method to couple different element topologies. The last example, a laser weld geometry, is employed to demonstrate the performance and scalability of our parallel implementation of the method, as well as coupling of more than two subdomains. Throughout our numerical examples, we omit the use of units unless they are necessary for the understanding of the problem at hand.

\subsection{Implementation}

The four variants of the Schwarz alternating method described in Section 2.4 have been implemented in a one-dimensional MATLAB code. The objective is to determine the convergence behavior, efficiency, and performance of each variant. This code has been optimized both in terms of memory usage and execution speed.

In addition, the Modified variant of the Schwarz alternating method described in Section 2.4 has been implemented in ALBANY, an open-source multiphysics research platform developed mainly at Sandia National

\footnotetext{
${ }^{3}$ See Remark 9 in the Appendix for a definition of geometric convergence.
} 
Laboratories. ${ }^{4}$ ALBANY is a C++ object-oriented, parallel, unstructured-grid, implicit finite element code for solving general partial differential equations. Among its salient features are:

- Use of components in code design for rapid development of capabilities [35].

- Extensive use of libraries from the open-source TRILINOS [33] project. $^{5}$

- Use of the TRILinos PhALANX package, specifically designed to solve general partial differential equations, that decomposes a complex problem into a number of simpler problems with managed dependencies.

- Use of the TRILINOS SACADO package for automatic differentiation. Thus, the stiffness blocks shown in (31) or (34) need not be derived and implemented explicitly. Their analytic values are computed automatically by the code, without resorting to numerical techniques such as finite differences.

ALBANY hosts a number of science and engineering applications, including the AERAS global atmosphere code [59], the Finite Elements for Land Ice eXperiments (FELIX) [63] ice sheet model solver, the Quantum Computer Aided Design (QCAD) [25] simulator, and the Laboratory for Computational Mechanics (LCM) [62] research code. This last project is specifically targeted at solid mechanics applications, and our implementation of the Schwarz alternating method is part of it. For a more detailed description of ALBANY and the physics implemented therein, the reader is referred to Salinger et al. [53].

An attractive feature of the block implementation of the Modified Schwarz alternating method detailed in Section 2.4 is that it fits more or less seamlessly into existing codes. For this reason, this is the only variant of the method that is currently implemented in ALBANY. Given an existing code for solving the problem of interest on a single domain, the following modifications are necessary to introduce the Modified Schwarz method:

1. An implementation of the projection operator $P_{\Omega_{i} \rightarrow \Gamma_{j}}[\cdot]$ in the form of the projection matrices (27) for the enforcement the Schwarz boundary conditions on $\Gamma_{i}$.

2. A modification to the calculation of the stiffness matrix and load vector, such that the block system in (31) is formed in place of a single subdomain linear system in the Newton method.

The computation of the projection $P_{\Omega_{i} \rightarrow \Gamma_{j}}[\cdot]$ embodied by the matrices (27) requires a search to determine the location of the nodes in $\Gamma_{j}$ within $\Omega_{i}$. The implementation of this search is more or less straightforward in single-processor computers, but more difficult for multi-processor computers. To perform the search in parallel for multi-processor computers, it is possible to adapt algorithms in some previously developed libraries, e.g., Hansen et al. [32] or Slattery, Wilson, and Pawlowski [56]. Our ALBANY implementation uses the latter of these algorithms, implemented in the Data Transfer Kit (DTK) [56], an open-source software library designed to provide parallel, scalable services for solution transfer between shared volumes and surfaces. ${ }^{6}$ For solution of the block linear system (31), we use the TRILINOS preconditioner package TEKO [16]. In addition, the three-dimensional geometries considered in Sections 4.4-4.6 were created using the CUBIT mesh generation toolkit [36].

\footnotetext{
${ }^{4}$ ALBANY is available on GitHub: https://github.com/gahansen/Albany

${ }^{5}$ TRILINOS is available on GitHub: https://github.com/trilinos/trilinos

${ }^{6}$ DTK is available on GitHub: https://github.com/ORNL-CEES/DataTransferKit
} 


\subsection{Error Analysis}

The numerical results presented below include an error analysis. Errors are evaluated in the discrete $l^{2}$ norm. In particular, the absolute $\epsilon_{\text {abs }}$ and relative $\epsilon_{\text {rel }}$ errors in a computed solution are calculated as

$$
\epsilon_{\mathrm{abs}}:=\left\|\hat{\boldsymbol{y}}_{\mathrm{nq}}-\boldsymbol{y}_{\mathrm{nq}}\right\|, \quad \epsilon_{\mathrm{rel}}:=\frac{\left\|\hat{\boldsymbol{y}}_{\mathrm{nq}}-\boldsymbol{y}_{\mathrm{nq}}\right\|}{\left\|\boldsymbol{y}_{\mathrm{nq}}\right\|},
$$

where $\|\cdot\|$ denotes the discrete $l^{2}$ norm, $\boldsymbol{y}_{\mathrm{nq}}$ is an exact or reference solution, and $\hat{\boldsymbol{y}}_{\mathrm{nq}}$ is the numerically computed solution. The vectors $\boldsymbol{y}_{\mathrm{nq}}$ and $\hat{\boldsymbol{y}}_{\mathrm{nq}}$ can be defined at nodes (e.g., for displacements) or quadrature points (e.g., for Cauchy stresses), and hence the subscript "nq".

All of our test cases are in the finite-deformation regime, for which a closed-form analytic solution is not available. Hence, we calculate the errors in (41) with respect to a reference solution, computed on a single domain discretized with a sufficiently fine mesh. In general, the reference geometry mesh and subdomain geometry meshes will be nonconforming; nevertheless, the errors (41) assume that the vectors $\hat{\boldsymbol{y}}_{\mathrm{nq}}$ and $\boldsymbol{y}_{\mathrm{nq}}$ have the same length and that their entries are defined at the same locations. To ensure this, we perform projections employing the DTK package, mentioned above in Section 4.1. The procedure is as follows. Let $\Omega_{\text {ref }}$ denote a high-resolution reference domain on which a reference nodal or quadrature point vector $\boldsymbol{y}_{\text {ref }}$ is computed. It is possible to use DTK to project $\boldsymbol{y}_{\text {ref }}$ onto an arbitrary domain $\Omega \subset \Omega_{\text {ref }}$ where we wish to compute the errors. Denoting this projection from $\Omega_{\text {ref }}$ to $\Omega$ by $P_{\Omega_{\text {ref }} \rightarrow \Omega \text {, we have that }}$

$$
\boldsymbol{y}_{\mathrm{nq}}:=P_{\Omega_{\mathrm{ref}} \rightarrow \Omega}\left[\boldsymbol{y}_{\mathrm{ref}}\right] .
$$

Once $\boldsymbol{y}_{\mathrm{nq}}$ has been computed using (42), we can use (41) to calculate the absolute or relative errors for a quantity of interest in a subdomain. The projection (42) is performed using an ALBANY utility created specifically for this purpose.

\subsection{Singular Bar}

The main objective of this example is to investigate the behavior of the four Schwarz variants of Section 2.4 described in more detail in Algorithms 2 to 5. The singular bar problem devised by Foulk [24] was used as a benchmark by Sun and Mota [61] for investigating multiscale coupling. Consider a one-dimensional bar with cross-section area $A(X)=A_{0} \sqrt{X / L}$, clamped at $X=0$, and with a prescribed displacement $\Delta$ at the end $X=L$, where $L$ is the length of the bar as shown in Figure 2 .

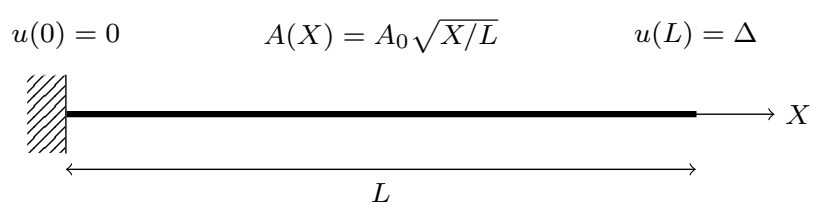

Figure 2: Schematic of the one-dimensional singular bar. This problem is well-posed despite the singularity at the left end provided that the stored energy is convex. See Appendix B for the analytic solution of the singular bar for the simplified case of linear elasticity.

Remarkably, the singular bar problem is well-posed despite the singularity at the left end, provided that the axial stress derives from a convex potential [29]. This well-posedness together with the strain singularity at the left end make the singular bar well-suited for the study of the behavior of methods under strain localization. See Appendix B for the analytic solution of a linear-elastic singular bar. 
Nevertheless, to exercise large elastic deformations in our examples, we choose a compressible NeoHookean-type energy density $W$. In the particular case of one-dimensional problems this energy density may be written as

$$
W[\lambda(X)]=\frac{1}{2} E\left\{[\lambda(X)]^{2}+[\lambda(X)]^{-2}-2\right\},
$$

where $\lambda(X):=d x(X) / d X$ is the stretch, $x(X)=X+u(X)$ is the position of a material point in the current configuration, $u(X)$ is the displacement field, and $E$ is the elastic modulus. The corresponding axial stress can then be written as

$$
\sigma(\lambda):=\frac{\partial W}{\partial \lambda}=E\left(\lambda-\lambda^{-3}\right) .
$$

We set the length of the bar to $L=1$, the reference cross-section area to $A_{0}=1$, the Young's modulus to $E=1$, and the applied displacement to $\Delta=1$ to double the length of the bar. For the application of the Schwarz alternating method, we decompose the domain into two subdomains, $\Omega_{1}$ and $\Omega_{2}$. In our first calculation, each subdomain is discretized using 48 elements with $50 \%$ overlap. Figure 3 shows convergence of the Full Schwarz method of Algorithm 2 to the single-domain solution as the iterations proceed in the Schwarz loop. The convergence of the Full Schwarz method with respect to the size of the overlap region for different mesh sizes is shown in Figure 4. As expected from the proof of convergence of Appendix A, the method does not converge for $0 \%$ overlap. Also as expected, faster convergence is observed as the size of the overlap region is increased. The other three methods, namely Modified Schwarz, Inexact Schwarz, and Monolithic Schwarz of Algorithms 3 to 5 show similar behavior.

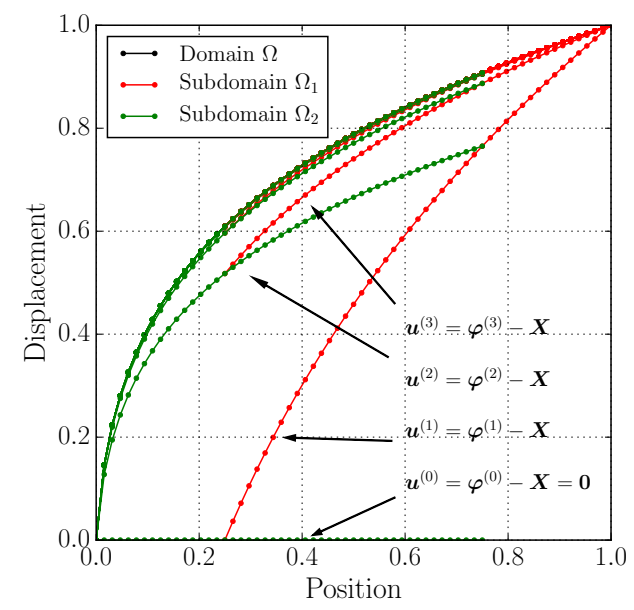

Figure 3: Full Schwarz method of Algorithm 2 applied to the singular bar problem discretized using two subdomains each having 48 elements and $50 \%$ overlap. The displacement field for subdomain $\Omega_{1}$ and $\Omega_{2}$ is shown in red and green respectively as the Schwarz iterations proceed. A conventional single-domain solution for 64 elements is plotted in black, but is covered by the red and green curves (and therefore invisible) as the Schwarz iterations converge to the single-domain solution. The other three methods of Algorithms 3 to 5 show similar behavior.

Next we investigate the convergence behavior of the four algorithms of Section 2.4. In keeping with the convergence criterion for the Schwarz loop proposed for Algorithms 2-5, we use the error measure for each 


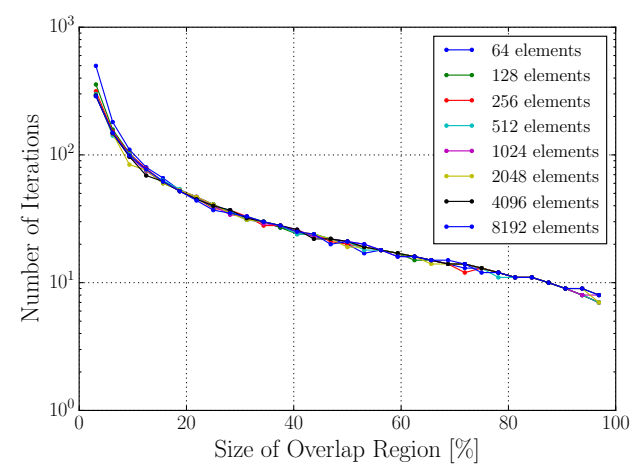

Figure 4: Convergence of the Full Schwarz method for the singular bar problem with respect to the size of the overlap region for different mesh sizes. The method does not converge for $0 \%$ overlap. If the overlap is $100 \%$ then the single-domain solution is recovered for each of the subdomains. The other three methods of Algorithms 3 to 5 show similar behavior.

Schwarz iteration

$$
\epsilon^{(n)}:=\left[\left(\frac{\left\|\triangle \boldsymbol{x}_{B}^{(n)}\right\|}{\left\|\boldsymbol{x}_{B}^{(n)}\right\|}\right)^{2}+\left(\frac{\left\|\triangle \boldsymbol{x}_{B}^{(n+1)}\right\|}{\left\|\boldsymbol{x}_{B}^{(n+1)}\right\|}\right)^{2}\right]^{1 / 2}
$$

for $n \in\{0,2,4, \ldots\}$. In our calculations, we find that the Full Schwarz, Inexact Schwarz and Monolithic Schwarz methods always converge monotonically. While the Modified Schwarz method converges monotonically most of the time, it ocassionally exhibits non-monotonic convergence. This is shown in Figure 5.

Lastly for the singular bar problem, we show a comparison of the time complexity of the four algorithms of Section 2.4 in Figure 6. The Modified Schwarz, Inexact Schwarz, and Monolithic Schwarz methods show remarkably similar time complexity across a variety of mesh sizes. The only significant difference that we observe is in the performance of the Full Schwarz method, which is consistently about 3 or 4 times slower than the other three methods. The increase in run times for small meshes shown in Figure 6 is due to overhead in managing small arrays in our MATLAB implementation of the four methods. The Modified Schwarz method is the only one we implemented in ALBANY since it is the simplest of the four Algorithms 2 to 5 and its time complexity is similar to that of the Inexact Schwarz and Monolithic Schwarz methods.

\subsection{Cuboid}

The main objective of this example is to show the effect of the size of the overlap region on the convergence of the Schwarz alternating method. For this, we use our ALBANY implementation of the Modified Schwarz method of Algorithm 3. This three-dimensional simple example involves the coupling of two cuboids of square base, denoted by $\Omega_{1}$ and $\Omega_{2}$, discretized using 8-node hexahedral elements with different levels of refinement. Each cuboid has side of length $l=1.0$ in their base and they are placed in space such that together they form a larger cuboid of square base in the $x y$ plane and height parallel to the $z$ axis. The material model used for this problem has the stored energy density

$$
W(\boldsymbol{C})=\frac{1}{4} \kappa\left(J^{2}-2 \log J-1\right)+\frac{1}{2} \mu\left(J^{-2 / 3} \operatorname{tr} \boldsymbol{C}-3\right),
$$




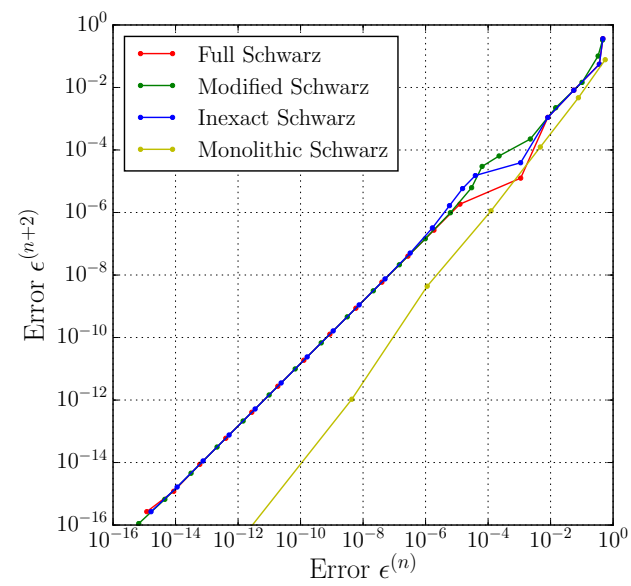

(a) 1024 elements per subdomain

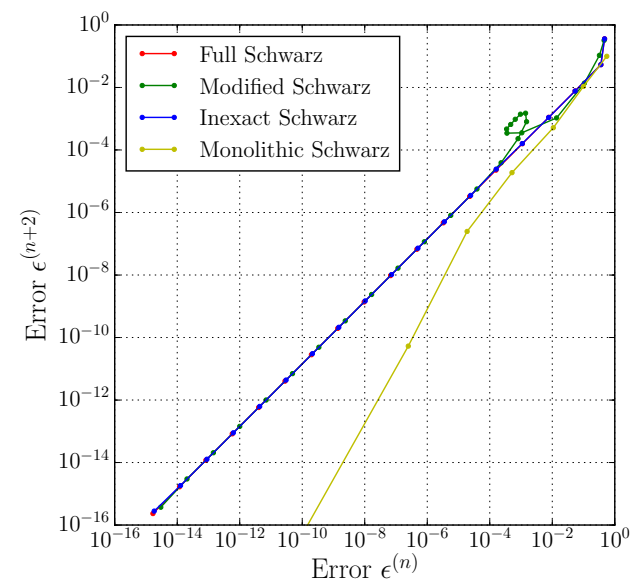

(b) 64 elements per subdomain

Figure 5: Convergence behavior of the four algorithms of Section 2.4 for the singular bar problem. The plotted error measure for the Schwarz iterations $\epsilon^{(n)}$ is defined in (45) in the notation of Algorithms 2-5. Occasionally, the Modified Schwarz method (green) displays non-monotonic convergence, as shown in the right plot. Numerical results reveal that the other three methods of Algorithms 3 to 5 always converge monotonically for this problem.

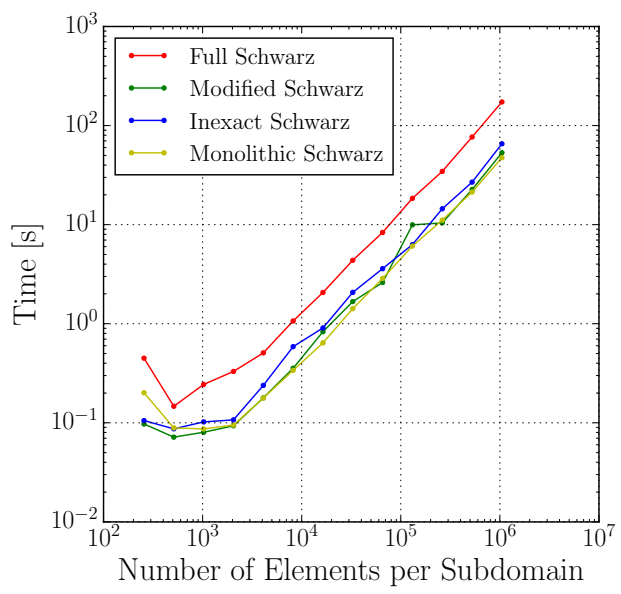

Figure 6: Time complexity of the four algorithms of Section 2.4 for the singular bar problem. The only significant difference is in the performance of the Full Schwarz method (red) with respect to the other three methods. Our MATLAB implementation of the four methods shows increase in run times for small meshes due to overhead in managing small arrays. 
where $\boldsymbol{C}:=\boldsymbol{F}^{\mathrm{T}} \boldsymbol{F}, J:=\operatorname{det} \boldsymbol{F}$, the bulk modulus $\kappa:=\frac{E}{3(1-2 \nu)}$, and the shear modulus $\mu:=\frac{E}{2(1+\nu)}$, in which the Young's modulus $E$ and Poisson's ratio $\nu$ are set to $E=1440$ and $\nu=0.25$. The boundary conditions are

$$
\begin{aligned}
u_{1}\left(X_{1}=0\right)=u_{2}\left(X_{2}=0\right)=u_{3}\left(X_{3}=0\right) & =0, \\
u_{3}\left(X_{3}=L\right) & =\Delta,
\end{aligned}
$$

where $L=2.0$ is the height of the entire cuboid, and $\Delta=10^{-2}$. A series of meshes are shown in Figure 7, where the domain $\Omega_{1}$ is discretized with a single element, and the domain $\Omega_{2}$ is discretized with 1,2 , 4 , and 8 element divisions per side. In addition to these discretizations, simulations are carried out with $h_{1} \in\left\{\frac{1}{2}, \frac{1}{4}, \frac{1}{8}\right\}$ for each value of $h_{2} \in\left\{1, \frac{1}{2}, \frac{1}{4}, \frac{1}{8}\right\}$. Moreover, the overlap volume fraction is varied for each pair $\left(h_{1}, h_{2}\right)$, such that the volume fraction of the overlap region $f_{\text {overlap }} \in\left\{\frac{1}{2}, \frac{1}{4}, \frac{1}{8}\right\}$.

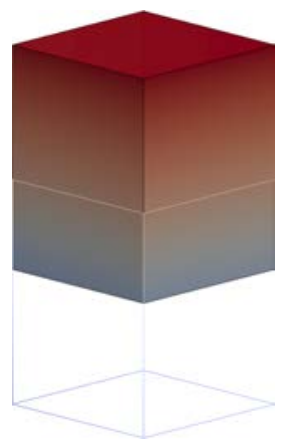

(a) $h_{1}=1, h_{2}=1$

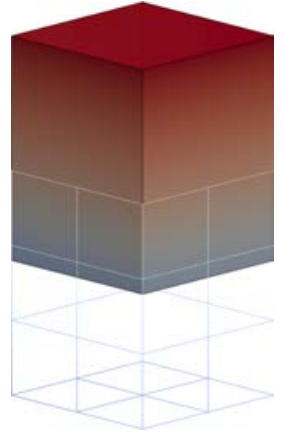

(b) $h_{1}=1, h_{2}=\frac{1}{2}$

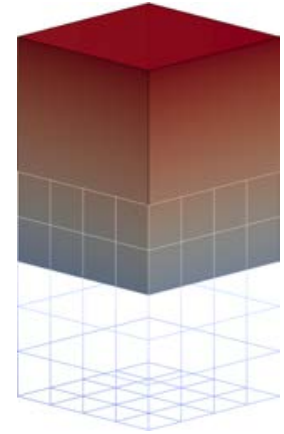

(c) $h_{1}=1, h_{2}=\frac{1}{4}$

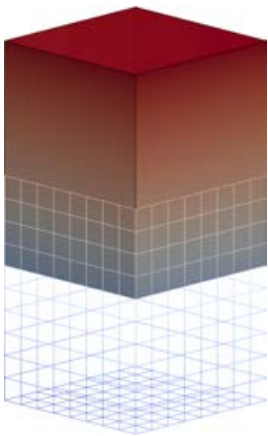

(d) $h_{1}=1, h_{2}=\frac{1}{8}$

Figure 7: Examples of discretization for the cuboid problem with element size $h_{1}=1$ in $\Omega_{1}$ and $h_{2} \in\left\{1, \frac{1}{2}, \frac{1}{4}, \frac{1}{8}\right\}$ in $\Omega_{2}$. The overlap volume fraction shown here is $f_{\text {overlap }}=\frac{1}{4}$.

The Modified Schwarz method of Algorithm 3 implemented in AlBANY has a single overall loop that combines the Newton and Schwarz iterations. Thus, with a slight abuse of notation, we will reuse the index $m$ for each of these iterations and relinquish the index $n$ for the cuboid example. This is done considering that keeping the index $n$ complicates notation in this setting. Therefore, for the convergence analysis, the magnitude of the increment $\Delta \boldsymbol{y}^{(m)}$ for the nodal displacements of the entire cuboid $\boldsymbol{y}$ at the Schwarz-Newton iteration $m$ is measured as

$$
\triangle \boldsymbol{y}^{(m)}=\left\|\boldsymbol{y}^{(m)}-\boldsymbol{y}^{(m-1)}\right\|
$$

for $m \in\{1,2, \ldots\}$. Then the rate of convergence $q$ is defined such that

$$
\triangle \boldsymbol{y}^{(m+1)} \leq \mu\left(\triangle \boldsymbol{y}^{(m)}\right)^{q}
$$

for $\mu \in(0,1)$. As it is shown in Figure 8, the convergence rate of the Modified Schwarz method is approximately linear $(q=1)$ for all the cases studied. The convergence factor $\mu$ is plotted as a function of overlap volume fraction in Figure 9. Here, it is seen that the convergence factor decreases with increasing overlap volume fraction, indicating faster linear convergence with increasing overlap volume fraction.

Next we turn our attention to the analysis of the error of the Modified Schwarz method for the coupled cuboid problem with respect to a relatively fine single-domain solution. In Table 1 we record the errors at the 


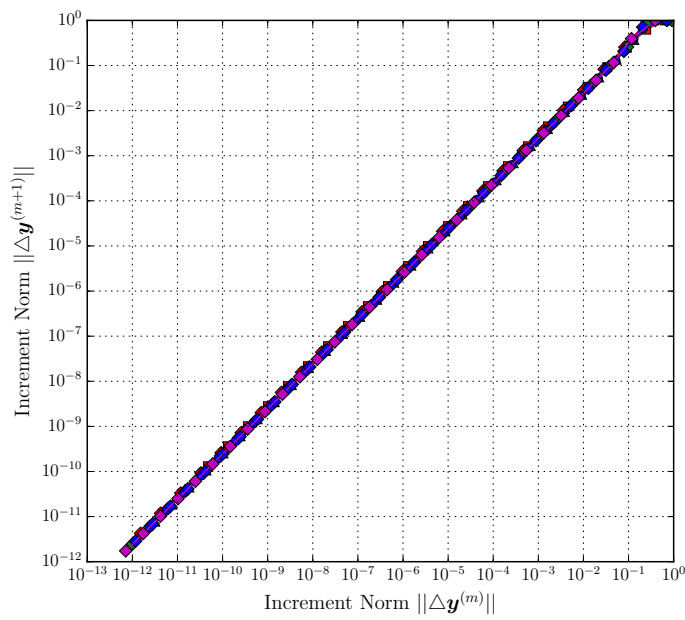

$$
\begin{aligned}
& \square\left(h_{1}, h_{2}\right)=(1,1) \\
& \Delta\left(h_{1}, h_{2}\right)=\left(1, \frac{1}{2}\right) \\
& \text { - }\left(h_{1}, h_{2}\right)=\left(1, \frac{1}{4}\right) \\
& \leadsto\left(h_{1}, h_{2}\right)=\left(1, \frac{1}{8}\right) \\
& \triangle \quad\left(h_{1}, h_{2}\right)=\left(\frac{1}{2}, \frac{1}{2}\right) \\
& \text { - }\left(h_{1}, h_{2}\right)=\left(\frac{1}{2}, \frac{1}{4}\right) \\
& \leadsto\left(h_{1}, h_{2}\right)=\left(\frac{1}{2}, \frac{1}{8}\right) \\
& \longrightarrow\left(h_{1}, h_{2}\right)=\left(\frac{1}{4}, \frac{1}{4}\right) \\
& \longleftrightarrow\left(h_{1}, h_{2}\right)=\left(\frac{1}{4}, \frac{1}{8}\right) \\
& \leadsto\left(h_{1}, h_{2}\right)=\left(\frac{1}{8}, \frac{1}{8}\right)
\end{aligned}
$$

Figure 8: Convergence of the cuboid problem for different mesh sizes and fixed overlap volume fraction $f_{\text {overlap }}=\frac{1}{4}$. The norm of the nodal displacement increment at the Schwarz-Newton iteration $m+1$ is plotted versus the norm of the increment at the Schwarz-Newton iteration $m$ from Algorithm 3. The convergence rate $q$ is given by the slope of the curves in the figure and for all cases it is approximately one.

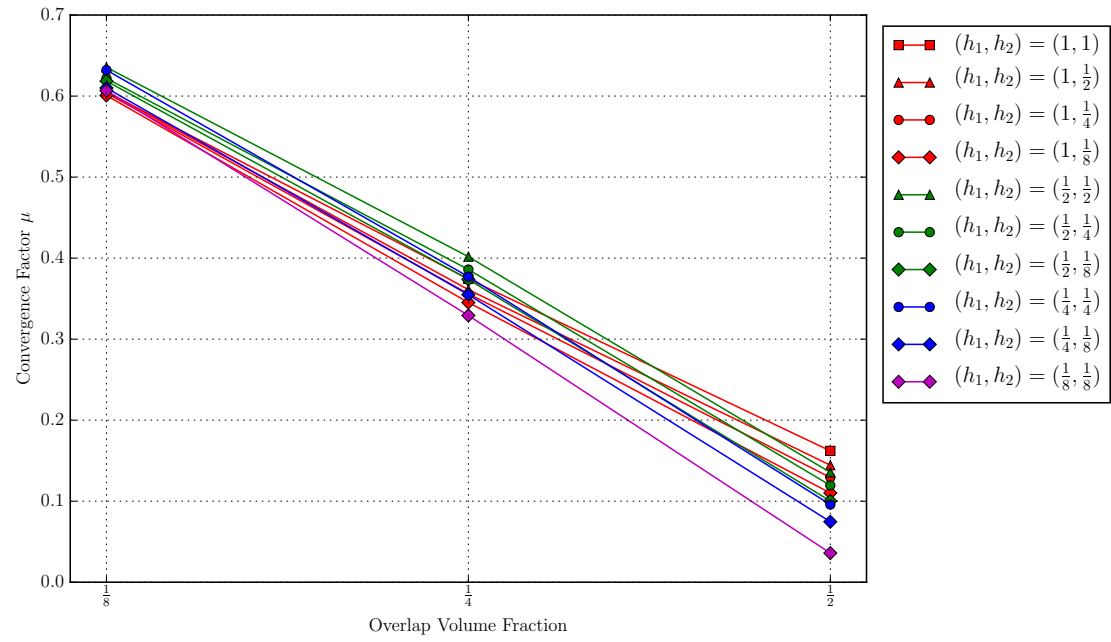

Figure 9: The convergence factor $\mu$ as a function of overlap volume and different mesh sizes for the cuboid problem. The figure shows monotonic decrease in the convergence factor with increasing overlap volume fraction for all the discretizations studied. 
end of the tenth applied load step for a case where the cuboids $\Omega_{1}$ and $\Omega_{2}$ were discretized using 96 and 12 hexahedral elements, respectively (Figure 10 (a) and (b)). The reference solution used for this error analysis was computed on a mesh (denoted by $\Omega_{\text {ref }}$ ) that has 128 hexahedral elements (Figure 10 (c)). The nonlinear solver tolerance for the norm of the residual when computing both the Schwarz and reference solutions was set to $\epsilon_{\mathrm{abs}}=10^{-12}$.

\begin{tabular}{ccc}
\hline Subdomain & $u_{3}$ relative error & $\sigma_{33}$ relative error \\
\hline$\Omega_{1}$ & $2.01 \times 10^{-13}$ & $2.45 \times 10^{-13}$ \\
$\Omega_{2}$ & $3.26 \times 10^{-13}$ & $5.10 \times 10^{-13}$ \\
\hline
\end{tabular}

Table 1: Relative errors in the displacement component $u_{3}$ and Cauchy stress component $\sigma_{33}$ for the cuboid problem after reaching the final applied displacement $\Delta=10^{-2}$ in 10 load steps. The errors were computed using the procedure and implementation described in Section 4.2.

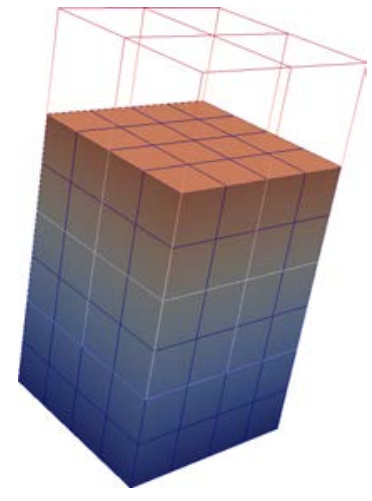

(a) $\Omega_{1}$

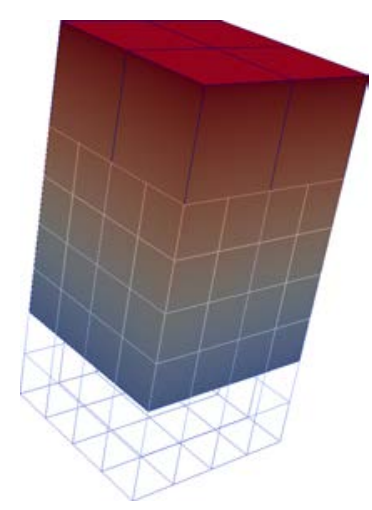

(b) $\Omega_{2}$
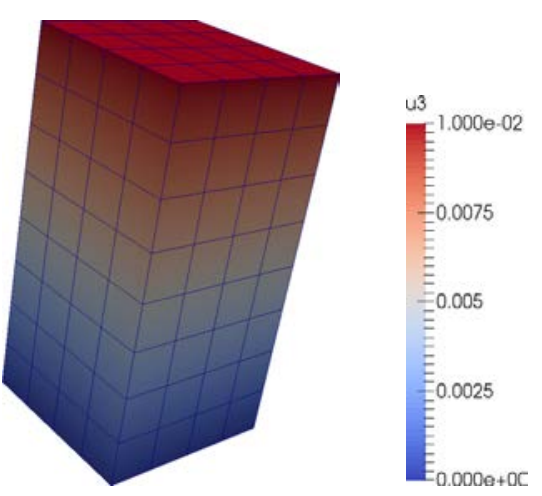

(c) $\Omega_{\mathrm{ref}}$

Figure 10: Comparison of the solution for the coupled cuboid problem with respect to a single-domain solution. The color scheme shows the displacement component $u_{3}$ at the final load step computed on $\Omega_{1}$ with 64 elements in (a) and $\Omega_{2}$ with 8 elements in (b). The reference single-domain solution on $\Omega_{\text {ref }}$ with 96 elements is shown in (c).

\subsection{Notched Cylinder}

In this example we aim to analyze the error due to coupling of meshes with different levels of refinement and element topologies. We consider a test case involving a notched cylinder that is stretched along its axial direction. A schematic of the geometry is shown in Figure 11 (a). The entire domain is denoted by $\Omega$, which is decomposed into two subdomains, $\Omega_{1}$ and $\Omega_{2}$, pictured in Figure 11 (c) and (d) in red and blue, respectively. We assume that the axis of the cylinder is parallel to the $z$ axis. Stress concentration and strain localization in the notch require a higher level of mesh refinement, and therefore it is natural to discretize $\Omega_{1}$, which contains the notch, with a finer mesh than $\Omega_{2}$.

The material model is the same Neohookean-type introduced in (46) for the cuboid problem, as well as 


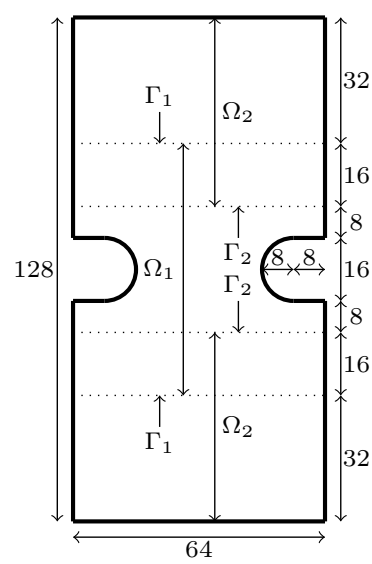

(a) Schematic

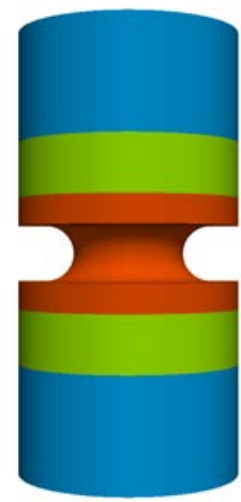

(b) Entire Domain $\Omega$

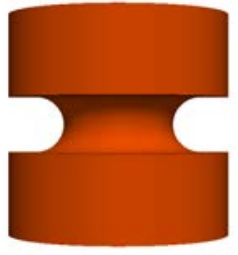

(c) Fine Region $\Omega_{1}$
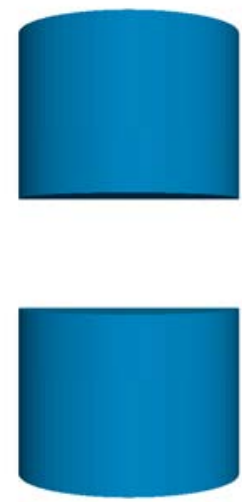

(d) Coarse Region $\Omega_{2}$

Figure 11: Domain decomposition of notched cylinder geometry $\Omega$ into a fine region $\Omega_{1}$ (red) and a coarse region $\Omega_{2}$ (blue). The overlap region is shown in green. Dimensions in $10^{-3}$.

the material properties $E=1440$ and $\nu=0.25$. We load the cylinder by using the boundary conditions

$$
u_{3}\left(X_{3}=-h / 2\right)=-\Delta, \quad u_{3}\left(X_{3}=h / 2\right)=\Delta
$$

where $h$ is the height of the cylinder, and $\Delta=6.4 \times 10^{-3}$.

\subsubsection{Conformal Hex-to-Hex Coupling}

First, we investigate the error introduced by the Schwarz method while removing other sources of error (e.g., geometric error) as much as possible. To this end, both subdomains, $\Omega_{1}$ and $\Omega_{2}$ are discretized using conformal hexahedral meshes (see Figure 12 (a) and (b), respectively). As shown in the figure, only one eighth of the cylinder is considered due to symmetry. The discretized subdomains $\Omega_{1}$ and $\Omega_{2}$ are comprised of $\sim 22,000$ and $\sim 40,000$ hexahedral elements, respectively. Figure 12 (c) shows a discretized reference domain containing $\sim 49,000$ hexahedral elements that will be denoted by $\Omega_{\text {ref }}$. By construction, the discretized domain $\Omega_{\text {ref }}$ is conformal with both $\Omega_{1}$ and $\Omega_{2}$. This is to ensure that any error in the coupled problem with respect to the reference solution in $\Omega_{\text {ref }}$ is due only to the Schwarz method and not to geometric or discretization mismatch.

In Table 2 we show the $l^{2}$ nodal relative error $\epsilon_{\mathrm{rel}}$ of (41) for the displacement component $u_{3}$ in $\Omega_{1}$ and $\Omega_{2}$ with respect to the reference solution computed on $\Omega_{\text {ref }}$ as a function of the nonlinear solver absolute tolerance on the residual. As this tolerance decreases to a value close to machine precision $\epsilon_{\text {machine }} \approx 2.2 \times 10^{-16}$, we observe that the error in the displacement decreases as well, approaching but not quite reaching machine precision. In this case, the only other source of error besides the Schwarz method itself is due to the interpolation performed by DTK.

Next, we examine the error in the Cauchy stress. In our initial error analysis, the stress was recovered in each of the subdomains by means of an $L^{2}$ projection. Figure 13 shows the error in the Cauchy stress component $\sigma_{33}$ with respect to the reference field in $\Omega_{\text {ref. }}$. The reader can observe from examining the figure that the error in the stress is negligible everywhere in $\Omega_{1}$ and $\Omega_{2}$ except at the boundaries $\Gamma_{1}$ and $\Gamma_{2}$. This 


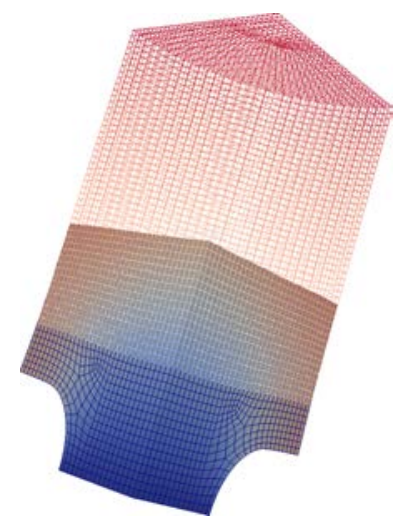

(a) $\Omega_{1}$

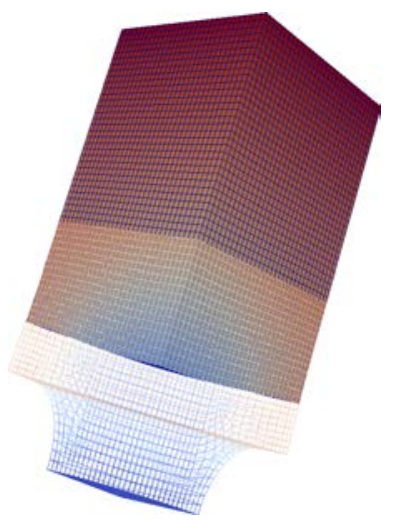

(b) $\Omega_{2}$

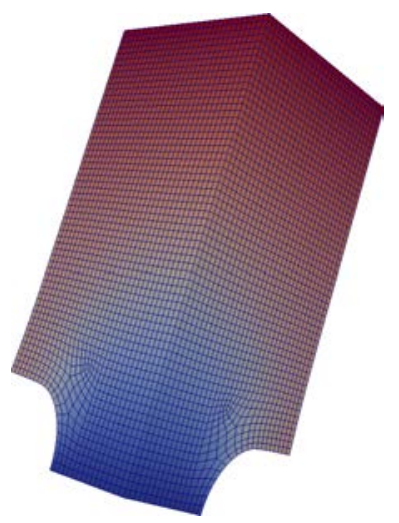

(c) $\Omega_{\text {ref }}$

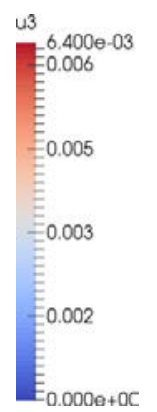

Figure 12: The displacement component $u_{3}$ at the final load step for the notched cylinder problem. Coupled solutions computed on conformal hexahedral meshes of $\Omega_{1}$ and $\Omega_{2}$ compared against reference solution on $\Omega_{\text {ref }}$.

\begin{tabular}{ccc}
\hline & \multicolumn{2}{c}{$u_{3}$ relative error } \\
Absolute residual tolerance & $\Omega_{1}$ & $\Omega_{2}$ \\
\hline $1.0 \times 10^{-4}$ & $7.60 \times 10^{-3}$ & $3.20 \times 10^{-3}$ \\
$1.0 \times 10^{-8}$ & $3.10 \times 10^{-5}$ & $1.71 \times 10^{-5}$ \\
$1.0 \times 10^{-12}$ & $1.34 \times 10^{-9}$ & $5.10 \times 10^{-10}$ \\
$1.0 \times 10^{-14}$ & $1.23 \times 10^{-11}$ & $4.69 \times 10^{-12}$ \\
$2.5 \times 10^{-16}$ & $1.14 \times 10^{-13}$ & $8.37 \times 10^{-14}$ \\
\hline
\end{tabular}

Table 2: Relative error for the nodal displacement component $u_{3}$ after 10 load steps for the notched cylinder problem in each of the subdomains for conformal hex-to-hex coupling. 
is due to the $L^{2}$ projection matrices for each of the subdomains lacking contributions from beyond the boundaries $\Gamma_{1}$ or $\Gamma_{2}$. Thus, even when the error in the displacement may be on the order of machine precision, it is important to emphasize that careful consideration should be given to stress recovery when dealing with any domain decomposition technique such as the Schwarz alternating method.

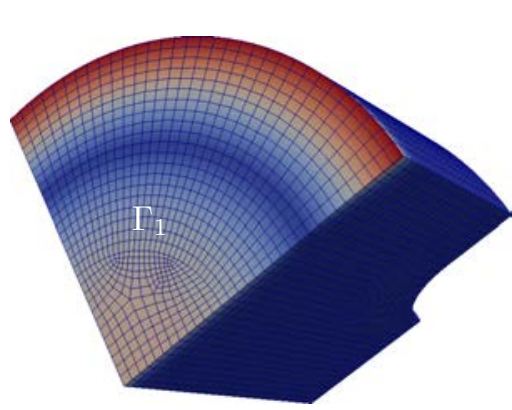

(a) $\Omega_{1}$

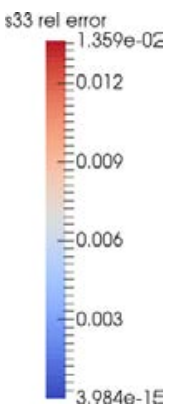

$.9840-16$

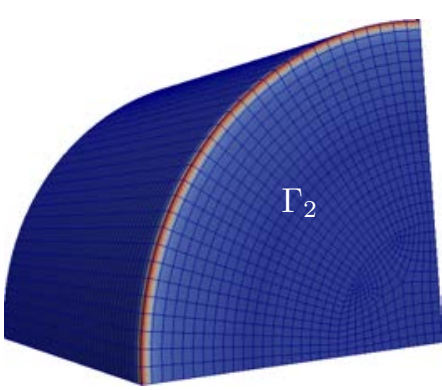

(c) $\Omega_{2}$

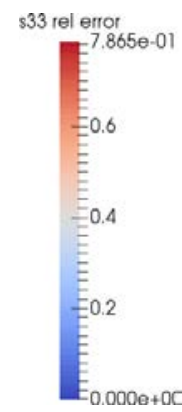

$0.0000+0 \mathrm{C}$

Figure 13: Relative errors for the Cauchy stress component $\sigma_{33}$ projected onto the nodes on $\Omega_{1}$ and $\Omega_{2}$ for conformal hex-to-hex coupling of the notched cylinder problem with respect to the reference solution on $\Omega_{\text {ref. }}$. The error is higher for $\Omega_{1}$ and $\Omega_{2}$ at the boundaries $\Gamma_{1}$ and $\Gamma_{2}$, respectively. This phenomenon is present in any domain decomposition technique and is not specific to the Schwarz alternating method.

To ascertain that the higher error in $\sigma_{33}$ on $\Gamma_{1}$ and $\Gamma_{2}$ shown in Figure 13 is indeed a consequence of the $L^{2}$ projection calculation, we calculate the relative error for the Cauchy stress component $\sigma_{33}$ on $\Gamma_{1}$ and $\Gamma_{2}$ averaged over quadrature points. These results are summarized in Table 3. As with the displacement component $u_{3}$ relative error, the stress $\sigma_{33}$ relative error becomes negligible as the absolute residual tolerance is decreased to machine precision.

\begin{tabular}{ccc}
\hline & \multicolumn{2}{c}{$\sigma_{33}$ relative error } \\
Absolute residual tolerance & $\Gamma_{1}$ & $\Gamma_{2}$ \\
\hline $1.0 \times 10^{-4}$ & $1.30 \times 10^{-2}$ & $4.38 \times 10^{-1}$ \\
$1.0 \times 10^{-8}$ & $5.31 \times 10^{-5}$ & $6.67 \times 10^{-4}$ \\
$1.0 \times 10^{-12}$ & $2.29 \times 10^{-9}$ & $2.89 \times 10^{-8}$ \\
$1.0 \times 10^{-14}$ & $2.11 \times 10^{-11}$ & $2.65 \times 10^{-10}$ \\
$2.5 \times 10^{-16}$ & $1.97 \times 10^{-13}$ & $1.98 \times 10^{-13}$ \\
\hline
\end{tabular}

Table 3: Relative error for the Cauchy stress component $\sigma_{33}$ on $\Gamma_{1}$ and $\Gamma_{2}$ with respect to reference solution on $\Omega_{\text {ref }}$ (averaged over quadrature points) for hex-to-hex conformal coupling for the notched cylinder problem. Figure 13 shows that stress recovery leads to some localized error at the Schwarz boundaries. Here we observe that averaging decreases the error significantly.

\subsubsection{Nonconformal Hex-to-Hex Coupling}

Next, we discretize the subdomains $\Omega_{1}$ and $\Omega_{2}$ with hexahedral nonconforming meshes as shown in Figure 14 (a) and (b). The meshes have $\sim 29,000$ and $\sim 47,000$ hexahedral elements, respectively. The reference domain $\Omega_{\text {ref }}$ is discretized with a hexahedral mesh that has $\sim 485,000$ hexahedral elements (Figure 14 (c)), 
and hence is substantially finer than the meshes for $\Omega_{1}$ and $\Omega_{2}$. For both the DTK search and error calculation, a substantially large point inclusion tolerance of 0.1 was used. ${ }^{7}$ Large values of this tolerance are needed when there is significant geometric mismatch between coupled meshes. This is specially relevant for curved geometries. Table 4 lists the nodal $l^{2}$ error $\epsilon_{\text {rel }}$ of (41) in the displacement component $u_{3}$ in $\Omega_{1}$ and $\Omega_{2}$ with respect to the reference solution computed on $\Omega_{\text {ref }}$ as a function of the nonlinear absolute residual tolerance. We observe that the relative errors remain near $1.30 \times 10^{-3}$ and $4.43 \times 10^{-4}$ for $\Omega_{1}$ and $\Omega_{2}$ respectively. This is due to irreducible geometric error, which can be seen by inspection of Figure 15.

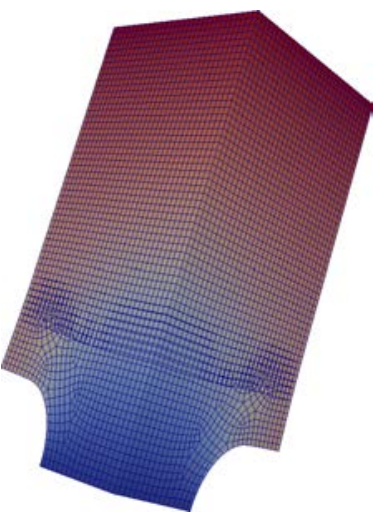

(a) $\Omega_{1}$ and $\Omega_{2}$

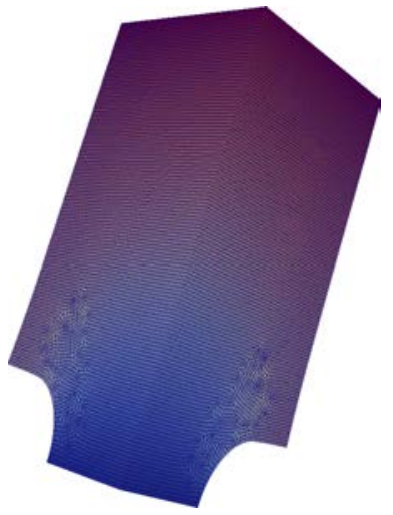

(b) $\Omega_{\text {ref }}$ mesh

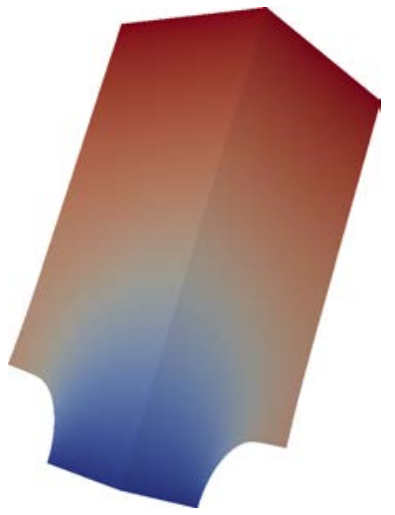

(c) $\Omega_{\text {ref }}$ solution

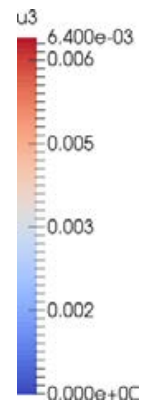

Figure 14: The displacement component $u_{3}$ at the final load step for the notched cylinder problem. Coupled solutions computed on nonconformal hexahedral meshes of $\Omega_{1}$ and $\Omega_{2}$ compared against reference solution on $\Omega_{\text {ref. }}$.

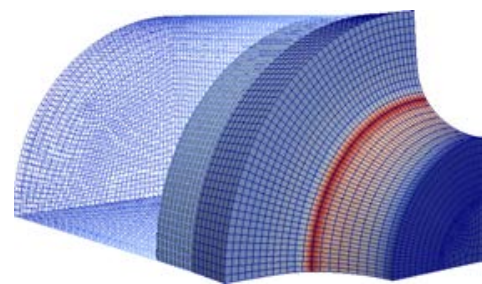

(a) $\Omega_{1}$

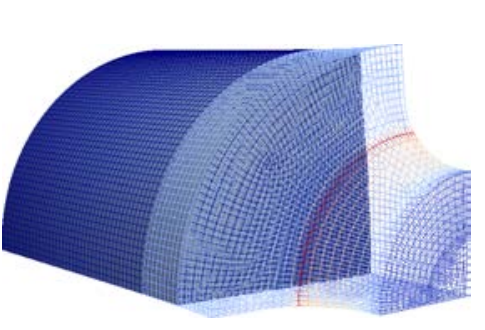

(b) $\Omega_{2}$

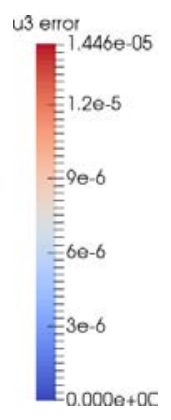

Figure 15: Absolute errors in the displacement component $u_{3}$ at the final load step for the notched cylinder problem. Error computed on coupled nonconformal hexahedral meshes of $\Omega_{1}$ and $\Omega_{2}$ with respect to the reference solution on $\Omega_{\text {ref }}$ Note that the error is highest where the curvature is highest, outside the overlapping region. This is an indication that the discretization error is more significant than any error introduced by the Schwarz coupling.

\footnotetext{
${ }^{7}$ The DTK point inclusion tolerance enlarges a target element so that source points outside of the element within the tolerance are considered to be inside of it. For a tolerance value of 0.1, elements in the target mesh are enlarged $10 \%$ in each dimension.
} 


\begin{tabular}{ccc}
\hline & \multicolumn{2}{c}{$u_{3}$ relative error } \\
Absolute residual tolerance & $\Omega_{1}$ & $\Omega_{2}$ \\
\hline $1.0 \times 10^{-8}$ & $1.31 \times 10^{-3}$ & $4.45 \times 10^{-4}$ \\
$1.0 \times 10^{-12}$ & $1.30 \times 10^{-3}$ & $4.43 \times 10^{-4}$ \\
$1.0 \times 10^{-14}$ & $1.30 \times 10^{-3}$ & $4.43 \times 10^{-4}$ \\
$2.5 \times 10^{-16}$ & $1.30 \times 10^{-3}$ & $4.43 \times 10^{-4}$ \\
\hline
\end{tabular}

Table 4: Relative error for the nodal displacement component $u_{3}$ after 10 load steps for the notched cylinder problem in each of the subdomains for nonconformal hex-to-hex coupling.

\subsubsection{Nonconformal Hex-to-Tet Coupling}

In the third instance of the notched cylinder example, the subdomain $\Omega_{1}$ is discretized using composite tetrahedral elements [45], whereas the subdomain $\Omega_{2}$ is discretized using hexahedral elements. The discretizations of these domains are shown in Figure 16 (a) and (b), respectively. The discretized subdomains $\Omega_{1}$ and $\Omega_{2}$ consist of only $\sim 4100$ and 90 elements respectively, therefore they are substantially coarser than the discretization of the reference domain $\Omega_{\text {ref }}$ that has $\sim 458,000$ elements (Figure 14 (c)). Table 5 records the nodal $l^{2}$ error $\epsilon_{\text {rel }}$ of (41) in the displacement component $u_{3}$ in $\Omega_{1}$ and $\Omega_{2}$ with respect to the reference solution computed on $\Omega_{\text {ref }}$. In this calculation, the nonlinear absolute residual tolerance was set to $\epsilon_{\mathrm{abs}}=10^{-14}$. The magnitudes of the errors recorded in Table 5 are expected considering the coarse mesh resolution and geometric error. As with the nonconformal hex-to-hex version of this problem, both the DTK search and error calculation use a point inclusion tolerance of 0.1 . This relatively high tolerance reflects the fact that two meshes of different element topologies and levels of refinement are used to resolve the curved boundary of the cylinder, which results in geometric mismatch. Figure 17 shows the absolute errors in the displacement component $u_{3}$ in $\Omega_{1}$ and $\Omega_{2}$ at the tenth load step. Although it is not as striking as in Figure 15, the irreducible geometric error is evident in this figure as well.

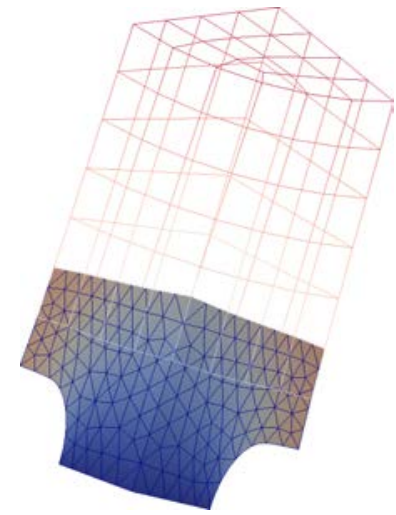

(a) $\Omega_{1}$

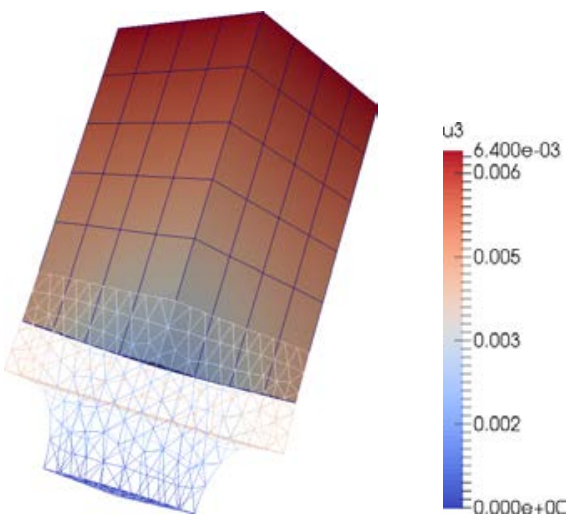

(b) $\Omega_{2}$

Figure 16: The displacement component $u_{3}$ at the final load step for the notched cylinder problem. Coupled solutions computed on composite tetrahedral [45] and hexahedral meshes of $\Omega_{1}$ and $\Omega_{2}$ respectively. 


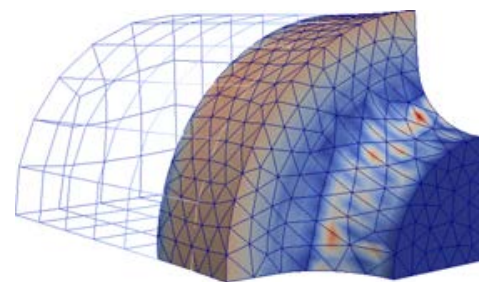

(a) $\Omega_{1}$

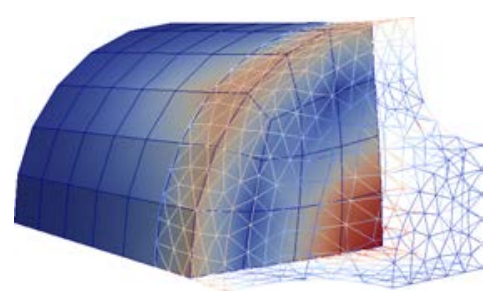

(b) $\Omega_{2}$

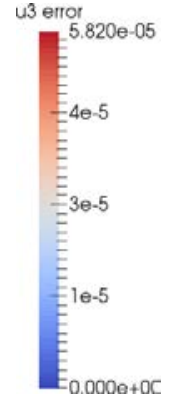

Figure 17: Absolute errors in the displacement component $u_{3}$ at the final load step for the notched cylinder problem. Error computed on coupled composite tetrahedral [45] and hexahedral meshes of $\Omega_{1}$ and $\Omega_{2}$ with respect to the reference solution on $\Omega_{\text {ref. }}$.

\begin{tabular}{ccc}
\hline & \multicolumn{2}{c}{$u_{3}$ relative error } \\
Absolute residual tolerance & $\Omega_{1}$ & $\Omega_{2}$ \\
\hline $1.0 \times 10^{-14}$ & $9.27 \times 10^{-3}$ & $3.70 \times 10^{-3}$ \\
\hline
\end{tabular}

Table 5: Relative error for the nodal displacement component $u_{3}$ after 10 load steps for the notched cylinder problem in each of the subdomains for hex-to-tet coupling.

For a case subject to larger deformation, Figure 18 shows the displacement component $u_{3}$ computed using our implementation of the Modified Schwarz method in ALBANY when we apply a larger prescribed displacement $\Delta=25.6 \times 10^{-3}$. This load is applied in a total of 1000 steps.

\subsection{Laser Weld}

Next, we investigate the strong scalability of the Schwarz alternating method on a problem and geometry intended to model a laser weld process. The domain $\Omega$ is decomposed into three subdomains: one subdomain representing the laser weld gauge (central region near the laser weld) and two subdomains representing holders on each side of the gauge (Figure 19). The fine and coarse subdomains are discretized as indicated in Figure 20. The material model used in these simulations is isotropic elasticity and $J_{2}$ plasticity with linear isotropic hardening. For the elasticity model, the Young's modulus is set to $10^{3}$, and the Poisson's ratio is 0.33 . For the $J_{2}$ plasticity model, the initial yield stress is given as $10^{1}$, and the hardening modulus is $10^{2}$. The ends of the specimen are subjected to clamped boundary conditions, and displaced \pm 0.3 symmetrically outward along the directions normal to the end faces.

For these calculations, we run the problem with a fixed mesh resolution on different numbers of processors. The scalability study was performed on the Sky Bridge Cray high-performance computing cluster located at Sandia National Laboratories. ${ }^{8}$ Figure 20 shows the scalability results for three instances of the laser weld problem for which ten load steps are applied. The total wall time for ALBANY in hours is plotted as a function of the number of processors on a log-log scale, along with a line of slope of -1 , which denotes perfect strong scaling. The reader can observe that the scalability is close to ideal for smaller numbers of processors, but

\footnotetext{
${ }^{8}$ https://www.top500.org/system/178518
} 


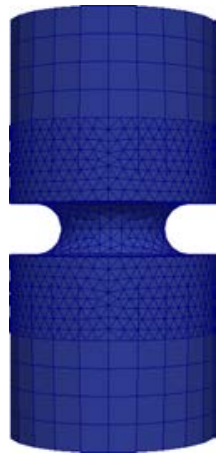

(a) Unloaded

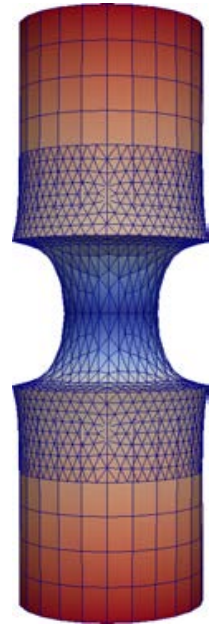

(b) Loaded

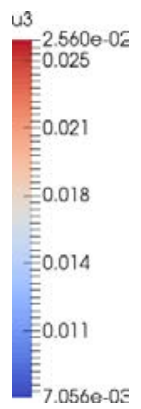

$7.0560-02$

Figure 18: The displacement component $u_{3}$ at the final load step for the notched cylinder problem stretched to a prescribed displacement of $\Delta=25.6 \times 10^{-3}$. Coupled solutions computed on composite tetrahedral [45] and hexahedral meshes for $\Omega_{1}$ and $\Omega_{2}$, respectively.

declines slightly for larger numbers of processors. This can be attributed in part to poor load balancing for the coarse meshes. In particular, remark that some of the coarse meshes have 80 elements, which can be distributed among at most 80 processors. Thus, when running on more than 80 processors, the resources allocated are not being utilized to their full potential. Figure 21 shows the deformed coarse and fine meshes together with the equivalent plastic strain. The plastic deformation for the most part is concentrated in the vicinity of the weld. In Figure 22, the contour of the error in the equivalent plastic strain in the fine region is plotted. The error is concentrated in the overlap region, where the maximum error is $5.4 \times 10^{-4}$. It is noted that the error is much lower in the critical region around the weld, as desired.

In case of very large deformation due to strain localization, re-meshing may be required to preserve the quality of the solution. For material models with internal states, this involves the mapping of internal variables from one mesh to another [43]. Within the context of the Schwarz alternating method, the re-meshing is effected only in the subdomains that need it. No other additional procedures are necessary as the method is indifferent to the level of refinement and element topologies of the different subdomains.

\section{Conclusions}

In this work we adopt the Schwarz alternating method as a coupling method for the finite-deformation solid mechanics problem. Our main contributions are:

1. A formal proof that the original method developed by Schwarz [55] (called herein Full Schwarz method) converges geometrically for the finite-deformation solid mechanics problem considered here. The proof is constructed by recourse to a variational formulation. To the best of our knowledge, we are the first to prove this result. 


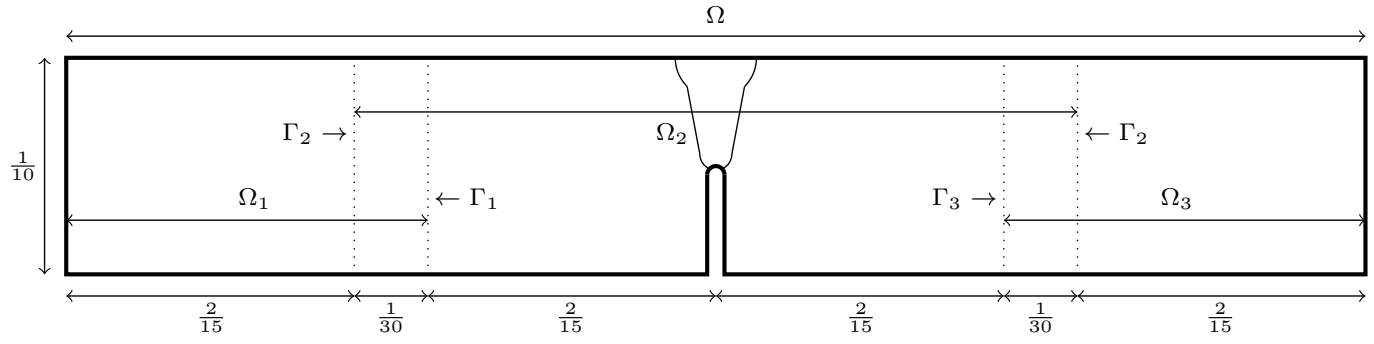

(a) Schematic of laser weld geometry.

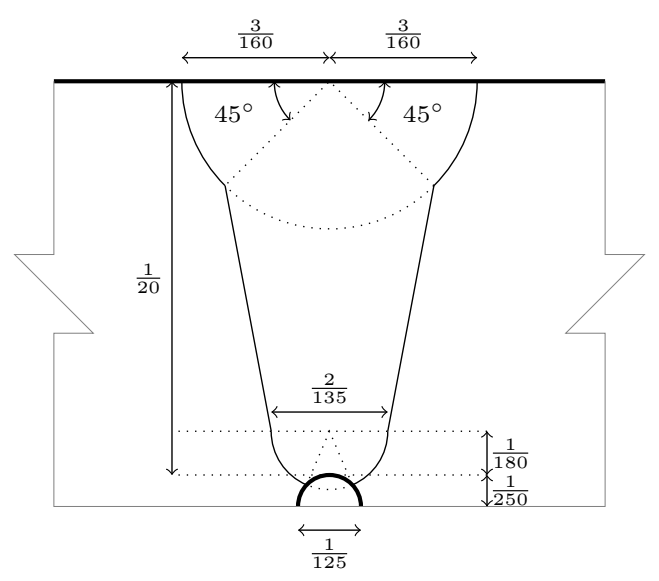

(b) Detail of the laser weld geometry.

Figure 19: Domain decomposition of the laser weld geometry $\Omega$ into one fine region $\Omega_{2}$ and two coarse regions $\Omega_{1}$ and $\Omega_{3}$ : (a) overall geometry, (b) laser weld detail. 


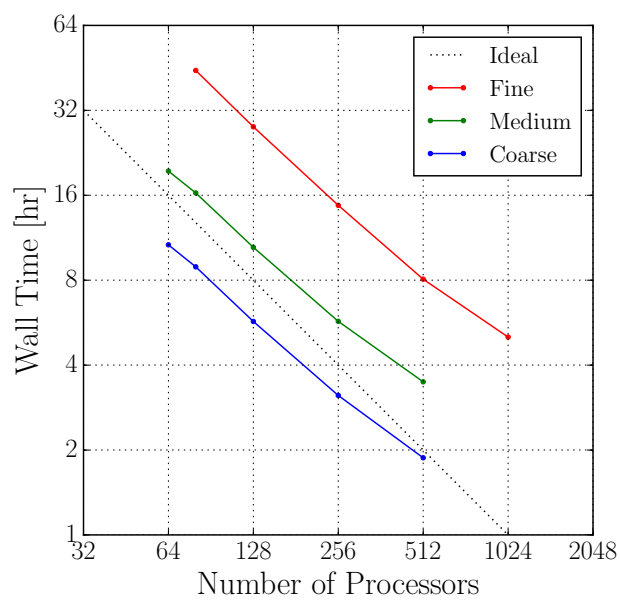

Figure 20: Strong scalability of the Schwarz alternating method for the laser weld problem with three levels of mesh refinement. The red curve is for coarse meshes of 1600 elements and a fine mesh of 114,804 elements. The green curve is for coarse meshes of 1600 elements and a fine mesh of 65,394 elements. The blue curve is for coarse meshes of 80 elements and a fine mesh of 65,394 elements. The black dotted line represents ideal strong scalability. The scalability is close to ideal for the three cases shown here.

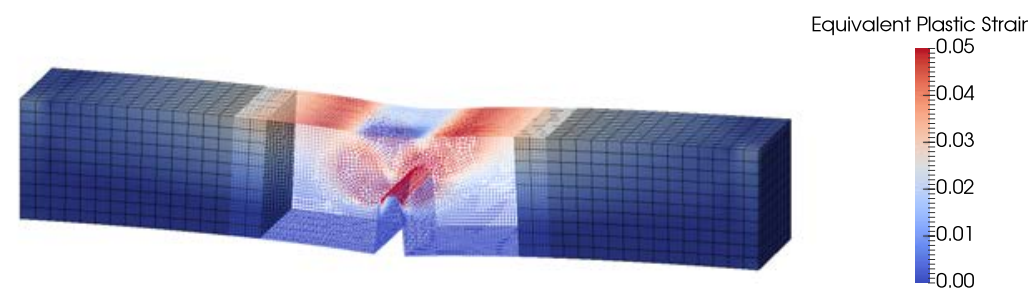

Figure 21: Deformed coarse and fine meshes together with the equivalent plastic strain for the laser weld specimen. The plastic deformation is concentrated mostly at the top of the notch. 


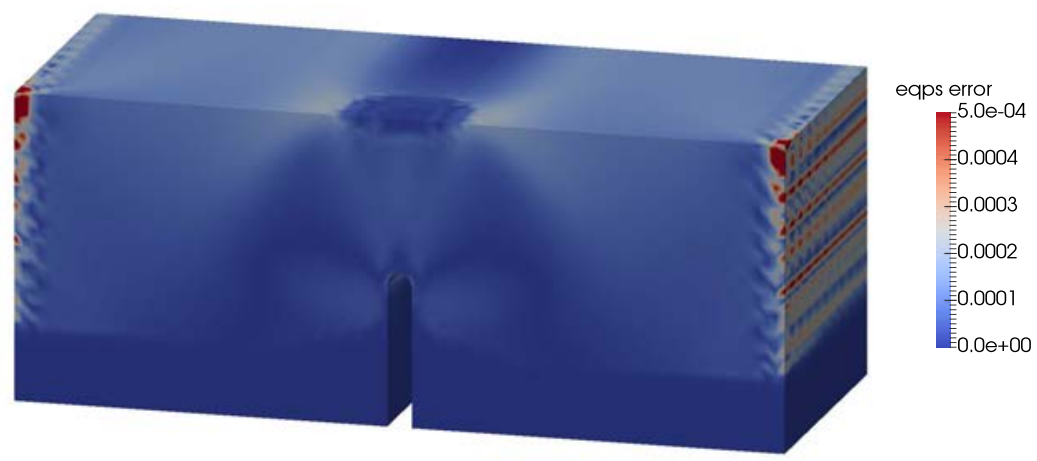

Figure 22: Contour of relative error in the equivalent plastic strain plotted on the deformed fine mesh. The error is mainly concentrated in the overlap region, where it reaches a maximum of $5.4 \times 10^{-4}$.

2. The development and introduction of four variants of the Schwarz alternating method and explicit algorithms for their implementation. The choice of one variant over another depends on the existing infrastructure of a computer implementation.

3. Demonstration by means of numerical examples that the convergence of the Schwarz method in its four variants is for the most part linear. The numerical examples revealed that the Modified Schwarz method occasionally displays non-monotonic behavior.

4. Demonstration of coupling of conformal meshes, non-conformal meshes, meshes with different levels of refinement, meshes with different element topologies, and more than two subdomains.

5. Demonstration that the error in the coupling can be decreased up to numerical precision provided that no other sources of error (such as geometric mismatch) exist.

6. The development of a parallel implementation of the Modified Schwarz method in the ALBANY code and demonstration that the strong scalability of our implementation is close to ideal.

Future work would include: extension of the methods presented herein to transient dynamics problems, including the ability to use different time steps and time integrators for each subdomain; development of a multi-physics coupling framework based on variational formulations and the Schwarz alternating method; analysis of the convergence for the other Schwarz variants introduced herein; investigation of the source of the non-monotonic behavior of the Modified Schwarz method; introduction of pervasive multithreading into our ALBANY implementation of the Schwarz alternating method using the KOKKOS framework [17].

\section{Acknowledgements}

Support for this work was received through the U.S. Department of Energy's (DOE) Advanced Simulation and Computing (ASC) Program at Sandia National Laboratories. Sandia National Laboratories is a multi-program 
laboratory managed and operated by Sandia Corporation, a wholly owned subsidiary of Lockheed Martin Corporation, for the U.S. Department of Energy's National Nuclear Security Administration under contract DE-AC04-94AL85000.

The writers gratefully acknowledge the many fruitful discussions they had in the development of this work with James Foulk, Glen Hansen, Jakob Ostien, and Andrew Salinger.

\section{A Proof of Convergence of the Schwarz Alternating Method for the Finite-Deformation Inelastic Problem}

In this section we give a proof of Theorem 1. The proof relies on several properties, presented below as remarks. Assume properties 1-5 enumerated in Section 3 hold.

Remark 1 By the coercivity of $\Phi[\varphi]$, it follows from the Lax-Milgram theorem that a unique minimizer to this functional over $\mathcal{S}$ exists, i.e., the minimization of $\Phi[\varphi]$ is well-posed.

Remark 2 By the Stampacchia theorem, the minimization of $\Phi[\varphi]$ in $\mathcal{S}$ is equivalent to finding $\varphi \in \mathcal{S}$ such that

$$
\left(\Phi^{\prime}[\varphi], \xi-\varphi\right) \geq 0
$$

for all $\xi \in \mathcal{S}$.

Remark 3 Recall that the strict convexity property of $\Phi[\varphi]$ can be written as

$$
\Phi\left[\boldsymbol{\psi}_{2}\right]-\Phi\left[\boldsymbol{\psi}_{1}\right]-\left(\Phi^{\prime}\left[\boldsymbol{\psi}_{1}\right], \boldsymbol{\psi}_{2}-\boldsymbol{\psi}_{1}\right) \geq 0,
$$

$\forall \boldsymbol{\psi}_{1}, \boldsymbol{\psi}_{2} \in \mathcal{S}$. From (36), remark that if $\Phi[\boldsymbol{\varphi}]$ is strictly convex over $\mathcal{S} \forall R \in \mathbb{R}$ such that $R<\infty$, we can find an $\alpha_{R}>0$ such that $\forall \boldsymbol{\psi}_{1}, \boldsymbol{\psi}_{2} \in \mathcal{K}_{R}$ we have

$$
\Phi\left[\boldsymbol{\psi}_{2}\right]-\Phi\left[\boldsymbol{\psi}_{1}\right]-\left(\Phi^{\prime}\left[\boldsymbol{\psi}_{1}\right], \boldsymbol{\psi}_{2}-\boldsymbol{\psi}_{1}\right) \geq \alpha_{R}\left\|\boldsymbol{\psi}_{2}-\boldsymbol{\psi}_{1}\right\|^{2} .
$$

Remark 4 By property 5 , the uniform continuity of $\Phi^{\prime}[\varphi]$, there exists a modulus of continuity $\omega>0$, with $\omega: \mathcal{K}_{R} \rightarrow \mathcal{K}_{R}$, such that

$$
\left\|\Phi^{\prime}\left(\boldsymbol{\psi}_{1}\right)-\Phi^{\prime}\left(\boldsymbol{\psi}_{2}\right)\right\| \leq \omega\left(\left\|\boldsymbol{\psi}_{1}-\boldsymbol{\psi}_{2}\right\|\right),
$$

$\forall \boldsymbol{\psi}_{1}, \boldsymbol{\psi}_{2} \in \mathcal{K}_{R}$. By definition, $\omega(\epsilon) \rightarrow 0$ as $\epsilon \rightarrow 0$.

Remark 5 It was shown in [38] that in the case $\Omega_{1} \cap \Omega_{2} \neq \emptyset, \forall \varphi \in \mathcal{S}$, there exist $\zeta_{1} \in \mathcal{S}_{1}$ and $\zeta_{2} \in \mathcal{S}_{2}$ such that

$$
\varphi=\zeta_{1}+\zeta_{2}
$$

and

$$
\max \left(\left\|\boldsymbol{\zeta}_{1}\right\|,\left\|\boldsymbol{\zeta}_{2}\right\|\right) \leq C_{0}\|\boldsymbol{\varphi}\|
$$

for some $C_{0}>0$ independent of $\varphi$.

Remark 6 Note that (39) can be written as

$$
\left(\Phi^{\prime}\left[\tilde{\boldsymbol{\varphi}}^{(n)}\right], \boldsymbol{\xi}^{(i)}\right)=0, \quad \text { for } \tilde{\boldsymbol{\varphi}}^{(n)} \in \tilde{\mathcal{S}}_{n}, \forall \boldsymbol{\xi}^{(i)} \in \mathcal{S}_{i},
$$

for $i \in\{1,2\}$ and $n \in\{0,1,2, \ldots\}$ (recall from (6) the relation between $i$ and $n$ ). This is due to the uniqueness of the solution to each minimization problem over $\tilde{\mathcal{S}}_{n}$ and the definition of $\tilde{\varphi}^{(n)}$ as the minimizer of $\Phi[\varphi]$ over $\tilde{\mathcal{S}}_{n}$. 
$\operatorname{Remark} 7$ Let $\tilde{\boldsymbol{\varphi}}^{(n)} \in \tilde{\mathcal{S}}_{n}$, and let $\boldsymbol{\xi} \in \mathcal{S}$. By Remark 5, there exist $\boldsymbol{\zeta}_{1} \in \mathcal{S}_{1}$ and $\boldsymbol{\zeta}_{2} \in \mathcal{S}_{2}$ such that

$$
\left(\Phi^{\prime}\left[\tilde{\boldsymbol{\varphi}}^{(n)}\right], \boldsymbol{\xi}\right)=\left(\Phi^{\prime}\left[\tilde{\boldsymbol{\varphi}}^{(n)}\right], \boldsymbol{\zeta}_{1}+\boldsymbol{\zeta}_{2}\right) .
$$

By (57), $\left(\Phi^{\prime}\left[\tilde{\boldsymbol{\varphi}}^{(n-1)}\right], \boldsymbol{\zeta}_{2}\right)=0$. Hence,

$$
\left(\Phi^{\prime}\left[\tilde{\boldsymbol{\varphi}}^{(n)}\right], \boldsymbol{\zeta}_{1}+\boldsymbol{\zeta}_{2}\right)=\left(\Phi^{\prime}\left[\tilde{\boldsymbol{\varphi}}^{(n)}\right], \boldsymbol{\zeta}_{1}+\boldsymbol{\zeta}_{2}\right)-\left(\Phi^{\prime}\left[\tilde{\boldsymbol{\varphi}}^{(n-1)}\right], \boldsymbol{\zeta}_{2}\right)=\left(\Phi^{\prime}\left[\tilde{\boldsymbol{\varphi}}^{(n)}\right], \boldsymbol{\zeta}_{2}\right)-\left(\Phi^{\prime}\left[\tilde{\boldsymbol{\varphi}}^{(n-1)}\right], \boldsymbol{\zeta}_{2}\right),
$$

since $\left(\Phi^{\prime}\left[\tilde{\varphi}^{(n)}\right], \zeta_{1}\right)=0$, also by (57). By the Cauchy-Schwarz inequality,

$$
\left(\Phi^{\prime}\left[\tilde{\boldsymbol{\varphi}}^{(n)}\right], \boldsymbol{\zeta}_{2}\right)-\left(\Phi^{\prime}\left[\tilde{\boldsymbol{\varphi}}^{(n-1)}\right], \boldsymbol{\zeta}_{2}\right)=\left(\Phi^{\prime}\left[\tilde{\boldsymbol{\varphi}}^{(n)}\right]-\Phi^{\prime}\left[\tilde{\boldsymbol{\varphi}}^{(n-1)}\right], \boldsymbol{\zeta}_{2}\right) \leq\left\|\Phi^{\prime}\left[\tilde{\boldsymbol{\varphi}}^{(n)}\right]-\Phi^{\prime}\left[\tilde{\boldsymbol{\varphi}}^{(n-1)}\right]\right\| \cdot\left\|\boldsymbol{\zeta}_{2}\right\| .
$$

Again using (57) and also (58) in (60) leads to

$$
\left(\Phi^{\prime}\left[\tilde{\boldsymbol{\varphi}}^{(n)}\right]-\Phi^{\prime}\left[\tilde{\boldsymbol{\varphi}}^{(n-1)}\right], \boldsymbol{\zeta}_{2}\right)=\left(\Phi^{\prime}\left[\tilde{\boldsymbol{\varphi}}^{(n)}\right], \boldsymbol{\xi}\right) \leq\left\|\Phi^{\prime}\left[\tilde{\boldsymbol{\varphi}}^{(n)}\right]-\Phi^{\prime}\left[\tilde{\boldsymbol{\varphi}}^{(n-1)}\right]\right\| \cdot\left\|\boldsymbol{\zeta}_{2}\right\|,
$$

and substituting (56) into (61) we finally obtain that

$$
\left(\Phi^{\prime}\left[\tilde{\boldsymbol{\varphi}}^{(n)}\right], \boldsymbol{\xi}\right) \leq C_{0}\left\|\Phi^{\prime}\left[\tilde{\boldsymbol{\varphi}}^{(n)}\right]-\Phi^{\prime}\left[\tilde{\boldsymbol{\varphi}}^{(n-1)}\right]\right\| \cdot\|\boldsymbol{\xi}\|,
$$

$\forall \boldsymbol{\xi} \in \mathcal{S}$.

Remark 8 For part (d) of Theorem 1, recall the definition of geometric convergence:

$$
E_{n+1} \leq C E_{n},
$$

$\forall n \in\{0,1,2, \ldots\}$ for some $C>0$, where

$$
E_{n}:=\left\|\tilde{\varphi}^{(n+1)}-\tilde{\varphi}^{(n)}\right\| .
$$

Remark 9 Recall from the definition of continuity that if $\Phi^{\prime}[\varphi]$ is Lipshitz continuous at $\tilde{\varphi}^{(n)}$ near $\varphi$, then there exists a constant $K \geq 0$ such that

$$
\frac{\left\|\Phi^{\prime}\left[\tilde{\varphi}^{(n)}\right]-\Phi^{\prime}[\varphi]\right\|}{\left\|\tilde{\varphi}^{(n)}-\varphi\right\|} \leq K
$$

Considering that $\Phi^{\prime}[\varphi]=\mathbf{0}$ since $\varphi$ is the minimizer of $\Phi[\varphi]$, (65) is equivalent to

$$
\left\|\Phi^{\prime}\left[\tilde{\varphi}^{(n)}\right]\right\| \leq K\left\|\tilde{\varphi}^{(n)}-\varphi\right\| .
$$

\section{Proof of Theorem 1}

Proof of $(a)$. Let $\tilde{\boldsymbol{\varphi}}^{(1)}=\arg \min _{\boldsymbol{\varphi} \in \tilde{\mathcal{S}}_{1}} \Phi[\boldsymbol{\varphi}]$. By (40), $\tilde{\boldsymbol{\varphi}}^{(1)} \in \tilde{\mathcal{S}}_{2}$. Let $\tilde{\boldsymbol{\varphi}}^{*}$ be the minimizer of $\Phi[\boldsymbol{\varphi}]$ over $\tilde{\mathcal{S}}_{2}$ and suppose $\Phi\left[\tilde{\varphi}^{*}\right]>\Phi\left[\tilde{\varphi}^{(1)}\right]$. But this is a contradiction, since we can take $\tilde{\varphi}^{*}=\tilde{\varphi}^{(1)}$. Hence, it cannot be that $\Phi\left[\tilde{\boldsymbol{\varphi}}^{(1)}\right]<\Phi\left[\tilde{\boldsymbol{\varphi}}^{(2)}\right]$ where $\tilde{\boldsymbol{\varphi}}^{(2)}=\arg \min _{\boldsymbol{\varphi} \in \tilde{\mathcal{S}}_{2}} \Phi[\boldsymbol{\varphi}]$. It follows by induction that

$$
\Phi\left[\tilde{\boldsymbol{\varphi}}^{(n)}\right] \leq \Phi\left[\tilde{\boldsymbol{\varphi}}^{(n-1)}\right]
$$

for $n \in\{1,2,3, \ldots\}$. Now let $\varphi$ be the minimizer of $\Phi[\varphi]$ over $\mathcal{S}$. Since the problem is well-posed $\varphi$ is unique. Hence $\Phi[\boldsymbol{\varphi}] \leq \Phi\left[\tilde{\varphi}^{(n)}\right]$ for all $n \in\{1,2,3, \ldots\}$. 
Proof of (b). By (a) $\Phi\left[\tilde{\varphi}^{(n)}\right] \rightarrow l$ as $n \rightarrow \infty$ for some $l \in \mathbb{R}$. Now, combining (51) and (53), we have the bound

$$
\Phi\left[\tilde{\boldsymbol{\varphi}}^{(n)}\right]-\Phi\left[\tilde{\boldsymbol{\varphi}}^{(n+1)}\right] \geq \Phi\left[\tilde{\boldsymbol{\varphi}}^{(n)}\right]-\Phi\left[\tilde{\boldsymbol{\varphi}}^{(n+1)}\right]-\left(\Phi^{\prime}\left[\tilde{\boldsymbol{\varphi}}^{(n+1)}\right], \tilde{\boldsymbol{\varphi}}^{(n)}-\tilde{\boldsymbol{\varphi}}^{(n+1)}\right) \geq \alpha_{R}\left\|\tilde{\boldsymbol{\varphi}}^{(n)}-\tilde{\boldsymbol{\varphi}}^{(n+1)}\right\|^{2},
$$

for all $n \in\{1,2,3, \ldots\}$. Since $\Phi\left[\tilde{\boldsymbol{\varphi}}^{(n)}\right] \rightarrow l$ as $n \rightarrow \infty$, it follows that $\Phi\left[\tilde{\boldsymbol{\varphi}}^{(n)}\right]-\Phi\left[\tilde{\boldsymbol{\varphi}}^{(n+1)}\right] \rightarrow 0$ as $n \rightarrow \infty$. From (68), we have that

$$
\lim _{n \rightarrow \infty}\left\|\tilde{\varphi}^{(n)}-\tilde{\varphi}^{(n+1)}\right\|^{2}=0
$$

from which we can conclude that $\tilde{\varphi}^{(n)}-\tilde{\varphi}^{(n+1)} \rightarrow \mathbf{0}$ as $n \rightarrow \infty$.

We must now show that $\tilde{\varphi}^{(n)}$ converges to $\varphi$, the minimizer of $\Phi[\varphi]$ on $\mathcal{S}$. By (53) with $\psi_{1}=\varphi$ and $\psi_{2}=\tilde{\varphi}^{(n)}$, we have

$$
\left\|\boldsymbol{\varphi}-\tilde{\boldsymbol{\varphi}}^{(n)}\right\|^{2} \leq \frac{1}{\alpha_{R}}\left\{\Phi[\boldsymbol{\varphi}]-\Phi\left[\tilde{\boldsymbol{\varphi}}^{(n)}\right]-\left(\Phi^{\prime}\left[\tilde{\boldsymbol{\varphi}}^{(n)}\right], \boldsymbol{\varphi}-\tilde{\boldsymbol{\varphi}}^{(n)}\right)\right\} .
$$

Since $\varphi$ is the mimimum of $\Phi[\varphi]$, by (a) we have that $\Phi[\varphi] \leq \Phi\left[\tilde{\varphi}^{(n)}\right]$. It follows that

$$
\Phi[\boldsymbol{\varphi}]-\Phi\left[\tilde{\boldsymbol{\varphi}}^{(n)}\right]-\left(\Phi^{\prime}\left[\tilde{\boldsymbol{\varphi}}^{(n)}\right], \boldsymbol{\varphi}-\tilde{\boldsymbol{\varphi}}^{(n)}\right) \leq-\left(\Phi^{\prime}\left[\tilde{\boldsymbol{\varphi}}^{(n)}\right], \boldsymbol{\varphi}-\tilde{\boldsymbol{\varphi}}^{(n)}\right)=\left(\Phi^{\prime}\left[\tilde{\boldsymbol{\varphi}}^{(n)}\right], \tilde{\boldsymbol{\varphi}}^{(n)}-\boldsymbol{\varphi}\right) .
$$

Subsituting (71) into (70) we have

$$
\left\|\boldsymbol{\varphi}-\tilde{\boldsymbol{\varphi}}^{(n)}\right\|^{2} \leq \frac{1}{\alpha_{R}}\left(\Phi^{\prime}\left[\tilde{\boldsymbol{\varphi}}^{(n)}\right], \tilde{\boldsymbol{\varphi}}^{(n)}-\boldsymbol{\varphi}\right) .
$$

Now by (62) (Remark 7),

$$
\left(\Phi^{\prime}\left[\tilde{\boldsymbol{\varphi}}^{(n)}\right], \tilde{\boldsymbol{\varphi}}^{(n)}-\boldsymbol{\varphi}\right) \leq C_{0}\left\|\Phi^{\prime}\left[\tilde{\boldsymbol{\varphi}}^{(n)}\right]-\Phi^{\prime}\left[\tilde{\boldsymbol{\varphi}}^{(n-1)}\right]\right\| \cdot\left\|\tilde{\boldsymbol{\varphi}}^{(n)}-\boldsymbol{\varphi}\right\| .
$$

Substituting (73) into (72) leads to

$$
\left\|\tilde{\boldsymbol{\varphi}}^{(n)}-\boldsymbol{\varphi}\right\| \leq \frac{C_{0}}{\alpha_{R}}\left\|\Phi^{\prime}\left[\tilde{\boldsymbol{\varphi}}^{(n)}\right]-\Phi^{\prime}\left[\tilde{\boldsymbol{\varphi}}^{(n-1)}\right]\right\| .
$$

Applying the uniform continuity assumption (54), we obtain

$$
\left\|\tilde{\varphi}^{(n)}-\boldsymbol{\varphi}\right\| \leq \frac{C_{0}}{\alpha_{R}} \omega\left(\left\|\tilde{\varphi}^{(n)}-\tilde{\varphi}^{(n-1)}\right\|\right) .
$$

By (69), $\left\|\tilde{\varphi}^{(n)}-\tilde{\varphi}^{(n-1)}\right\| \rightarrow 0$ as $n \rightarrow \infty$. From this we obtain the result, namely that $\tilde{\varphi}^{(n)} \rightarrow \varphi$ as $n \rightarrow \infty$.

Proof of (c). This follows immediately from (a) and (b).

Proof of $(d)$. By (b), for large enough $n$, there exists some $C_{1}>0$ independent of $n$ such that

$$
\left\|\tilde{\varphi}^{(n)}-\varphi\right\|^{2} \leq C_{1}\left\|\tilde{\varphi}^{(n+1)}-\tilde{\varphi}^{(n)}\right\|^{2} .
$$

Let us choose $C_{1}$ such that $C_{1}>\alpha_{R} / K$, where $K$ is the Lipshitz continuity constant in (66). Combining (68) with (76) leads to

$$
\frac{1}{\alpha_{R}}\left(\Phi\left[\tilde{\boldsymbol{\varphi}}^{(n)}\right]-\Phi\left[\tilde{\boldsymbol{\varphi}}^{(n+1)}\right]\right) \geq\left\|\tilde{\boldsymbol{\varphi}}^{(n+1)}-\tilde{\boldsymbol{\varphi}}^{(n)}\right\|^{2} \geq \frac{1}{C_{1}}\left\|\tilde{\boldsymbol{\varphi}}^{(n)}-\boldsymbol{\varphi}\right\|^{2} .
$$


Using the identity $\Phi\left[\tilde{\boldsymbol{\varphi}}^{(n)}\right]-\Phi\left[\tilde{\boldsymbol{\varphi}}^{(n+1)}\right]=\left(\Phi\left[\tilde{\boldsymbol{\varphi}}^{(n)}\right]-\Phi[\boldsymbol{\varphi}]\right)-\left(\Phi\left[\tilde{\boldsymbol{\varphi}}^{(n+1)}\right]-\Phi[\boldsymbol{\varphi}]\right)$, it follows from (77) that

$$
\left(\Phi\left[\tilde{\boldsymbol{\varphi}}^{(n)}\right]-\Phi[\boldsymbol{\varphi}]\right)-\left(\Phi\left[\tilde{\boldsymbol{\varphi}}^{(n+1)}\right]-\Phi[\boldsymbol{\varphi}]\right) \geq \frac{\alpha_{R}}{C_{1}}\left\|\tilde{\boldsymbol{\varphi}}^{(n)}-\boldsymbol{\varphi}\right\|^{2} .
$$

Substituting $\psi_{1}=\tilde{\varphi}^{(n)}$ and $\psi_{2}=\varphi$ into (53) and rearranging, we obtain

$$
\left(\Phi^{\prime}\left[\tilde{\boldsymbol{\varphi}}^{(n)}\right], \boldsymbol{\varphi}-\tilde{\boldsymbol{\varphi}}^{(n)}\right) \leq\left(\Phi^{\prime}\left[\tilde{\boldsymbol{\varphi}}^{(n)}\right], \boldsymbol{\varphi}-\tilde{\boldsymbol{\varphi}}^{(n)}\right)+\alpha_{R}\left\|\boldsymbol{\varphi}-\tilde{\boldsymbol{\varphi}}^{(n)}\right\| \leq \Phi[\boldsymbol{\varphi}]-\Phi\left[\tilde{\boldsymbol{\varphi}}^{(n)}\right]
$$

since $\alpha_{R} \geq 0$. Now, by the Cauchy-Schwarz inequality followed by the application of the Lipshitz continuity of $\Phi^{\prime}[\varphi](66)$ we can write

$$
\left(\Phi^{\prime}\left[\tilde{\boldsymbol{\varphi}}^{(n)}\right], \boldsymbol{\varphi}-\tilde{\boldsymbol{\varphi}}^{(n)}\right) \leq\left\|\Phi^{\prime}\left[\tilde{\boldsymbol{\varphi}}^{(n)}\right]\right\| \cdot\left\|\boldsymbol{\varphi}-\tilde{\boldsymbol{\varphi}}^{(n)}\right\| \leq K\left\|\boldsymbol{\varphi}-\tilde{\boldsymbol{\varphi}}^{(n)}\right\|^{2} .
$$

Hence, from (79),

$$
\Phi\left[\tilde{\varphi}^{(n)}\right]-\Phi[\varphi] \leq K\left\|\tilde{\varphi}^{(n)}-\varphi\right\|^{2} .
$$

Moreover, by (53) since $\Phi^{\prime}[\varphi]=\mathbf{0}$,

$$
\Phi\left[\tilde{\boldsymbol{\varphi}}^{(n)}\right]-\Phi[\boldsymbol{\varphi}] \geq \alpha_{R}\left\|\tilde{\boldsymbol{\varphi}}^{(n)}-\boldsymbol{\varphi}\right\|^{2} .
$$

Using (81) and (82) we obtain

$$
\left(\Phi\left[\tilde{\boldsymbol{\varphi}}^{(n)}\right]-\Phi[\boldsymbol{\varphi}]\right)-\left(\Phi\left[\tilde{\boldsymbol{\varphi}}^{(n+1)}\right]-\Phi[\boldsymbol{\varphi}]\right) \leq K\left\|\tilde{\boldsymbol{\varphi}}^{(n)}-\boldsymbol{\varphi}\right\|^{2}-\alpha_{R}\left\|\tilde{\boldsymbol{\varphi}}^{(n+1)}-\boldsymbol{\varphi}\right\|^{2} .
$$

Combining (83) and (78) leads to

$$
\frac{\alpha_{R}}{C_{1}}\left\|\tilde{\boldsymbol{\varphi}}^{(n)}-\boldsymbol{\varphi}\right\|^{2} \leq\left(\Phi\left[\tilde{\boldsymbol{\varphi}}^{(n)}\right]-\Phi[\boldsymbol{\varphi}]\right)-\left(\Phi\left[\tilde{\boldsymbol{\varphi}}^{(n+1)}\right]-\Phi[\boldsymbol{\varphi}]\right) \leq K\left\|\tilde{\boldsymbol{\varphi}}^{(n)}-\boldsymbol{\varphi}\right\|^{2}-\alpha_{R}\left\|\tilde{\boldsymbol{\varphi}}^{(n+1)}-\boldsymbol{\varphi}\right\|^{2},
$$

or

$$
\left\|\tilde{\varphi}^{(n+1)}-\varphi\right\| \leq B\left\|\tilde{\varphi}^{(n)}-\varphi\right\|
$$

with

$$
B:=\sqrt{\frac{K}{\alpha_{R}}-\frac{1}{C_{1}}}
$$

and $B \in \mathbb{R}$ as we chose $C_{1}>\alpha_{R} / K$. Furthermore, since the sequence $\left\{\tilde{\boldsymbol{\varphi}}^{(n)}\right\}$ converges monotonically to the minimizer $\varphi$ of $\Phi[\varphi]$ by (b) and (c), it follows that $B \in(0,1)$. Define $C:=1-B \in(0,1)$, then (85) can be recast as

$$
\left\|\tilde{\varphi}^{(n+1)}-\tilde{\varphi}^{(n)}\right\| \leq C\left\|\tilde{\varphi}^{(n)}-\tilde{\varphi}^{(n-1)}\right\|
$$

whereupon the claim is proven.

\section{B Analytic Solution for Linear-Elastic Singular Bar}

As reference, herein we provide the solution of the singular bar of Section 4.3 for linear elasticity. The equilibrium equation is

$$
P=\sigma(X) A(X)=\text { const. }, \quad \sigma(X)=E \epsilon(X), \quad \epsilon(X):=u^{\prime}(X), \quad A(X)=A_{0}\left(\frac{X}{L}\right)^{\frac{1}{2}}
$$


where $E$ is Young's modulus, $\sigma(X)$ is the axial stress, $\epsilon(X)$ is the axial strain and $u(X)$ is the displacement. Differentiating with respect to the referential coordinate $X$ we obtain

$$
[\sigma(X) A(X)]^{\prime}=0,
$$

which leads to the differential equation for the displacement

$$
\frac{1}{2 L}\left(\frac{X}{L}\right)^{-\frac{1}{2}} u^{\prime}(X)+\left(\frac{X}{L}\right)^{\frac{1}{2}} u^{\prime \prime}(X)=0, \quad u(0)=0, \quad u(L)=\Delta .
$$

Inspection of this equation leads to propose the ansatz for the displacement

$$
u(X)=\Delta\left(\frac{X}{L}\right)^{m}
$$

where the exponent $m$ is determined by direct substitution into (90), with the result

$$
u(X)=\Delta\left(\frac{X}{L}\right)^{\frac{1}{2}}
$$

together with the axial strain

$$
\epsilon(X)=\frac{\Delta}{2 L}\left(\frac{X}{L}\right)^{-\frac{1}{2}}
$$

which is singular at $X=0$ as desired.

\section{References}

[1] P. Aubertin, J. Réthoré, and R. de Borst. "Energy conservation of atomistic/continuum coupling". In: International Journal for Numerical Methods in Engineering 78.11 (2009), pp. 1365-1386. ISSN: 1097-0207.

[2] I. Babuška. "On the Schwarz algorithm in the theory of differential equations of mathematical physics (in Russian)". In: Chechoslovak Mathematical Journal 8.3 (1958), pp. 328-343.

[3] M. E. Backman and S.A. Finnegan. "The propagation of adiabatic shear". In: Metallurgical Effects of High Strain Rates 16 (1973), pp. 531-543.

[4] L. Badea. "On the Schwarz alternating method with more than two subdomains for nonlinear monotone problems". In: SIAM Journal on Numerical Analysis 28.1 (1991), pp. 179-204.

[5] P.T. Bauman, J.T. Oden, and S. Prudhomme. "Adaptive multiscale modeling of polymeric materials with Arlequin coupling and Goals algorithms". In: Computer Methods in Applied Mechanics and Engineering 198.5 (2009), pp. 799-818.

[6] P.T. Bauman et al. "On the application of the Arlequin method to the coupling of particle and continuum models". In: Computational Mechanics 42 (4 2008), pp. 511-530. ISSN: 0178-7675.

[7] F. Ben Belgacem. "The Mortar finite element method with Lagrange multipliers". English. In: $N U$ MERISCHE MATHEMATIK 84.2 (Dec. 1999), 173-197. ISSN: 0029-599X. 
[8] T. Belytschko, S. Loehnert, and J-H. Song. "Multiscale aggregating discontinuities: A method for circumventing loss of material stability". In: International Journal for Numerical Methods in Engineering 73.6 (2008), pp. 869-894. ISSN: 1097-0207.

[9] H. Ben Dhia. "Multiscale mechanical problems: the Arlequin method". In: Comptes Rendus de l'Academie des Sciences Series IIB Mechanics Physics Astronomy 326.12 (1998), pp. 899-904.

[10] H. Ben Dhia and G. Rateau. "The Arlequin method as a flexible engineering design tool". In: International Journal for Numerical Methods in Engineering 62.11 (2005), pp. 1442-1462. ISSN: 1097-0207.

[11] R.I. Borja. "A finite element model for strain localization analysis of strongly discontinuous fields based on standard Galerkin approximation". In: Computer Methods in Applied Mechanics and Engineering 190.11-12 (2000), pp. $1529-1549$.

[12] R.I. Borja. "Finite element simulation of strain localization with large deformation: capturing strong discontinuity using a Petrov-Galerkin multiscale formulation". In: Computer Methods in Applied Mechanics and Engineering 191.27-28 (2002), pp. $2949-2978$.

[13] M. Borrel, L. Halpern, and J. Ryan. "Euler/Navier-Stokes couplings for multiscale aeroacoustic problems". In: 20th AIAA Computational Fluid Dynamics Conference, Honolulu, Hawaii 2011-3047 (June 2011).

[14] J.H. Brunton et al. "The deformation of metals by high velocity impact". In: Metals For The Space Age, Plansee Proceedings 1964 (1965), pp. 137-148.

[15] M. Cervera, R. Codina, and M. Galindo. "On the computational efficiency and implementation of block-iterative algorithms for nonlinear coupled problems”. In: Engineering Computations 13.6 (1996), pp. 4-30.

[16] E.C. Cyr, J.N. Shadid, and R.S. Tuminaro. "Teko: A Block Preconditioning Capability with Concrete Example Applications in Navier-Stokes and MHD”. In: SIAM Journal of Scientific Computing (in press) (2016).

[17] I. Demeshko et al. "Towards Performance-Portability of the Albany Finite Element Analysis Code Using the Kokkos Library". In: International Journal of High Performance Computing Applications (2016).

[18] M. Engel and M. Griebel. "Flow simulation on moving boundary-fitted grids and application to fluidstructure interaction problems". In: Internation Journal for Numerical Methods in Fluids 50.4 (2005), pp. 437-468.

[19] D.J. Evans et al. "The convergence rate of the Schwarz alternating procedure (I): For one-dimensional problems”. In: International Journal for Computer Mathematics 20.2 (1986), pp. 157-170.

[20] D.J. Evans et al. "The convergence rate of the Schwarz alternating procedure (II): For two-dimensional problems". In: International Journal for Computer Mathematics 20.3-4 (1986), pp. 325-339.

[21] C. Farhat et al. "FETI-DP: a dual-primal unified FETI method - part I: A faster alternative to the two-level FETI method". English. In: INTERNATIONAL JOURNAL FOR NUMERICAL METHODS IN ENGINEERING 50.7 (Mar. 2001), 1523-1544. ISSN: 0029-5981.

[22] F. Feyel. "A multilevel finite element method (FE2) to describe the response of highly non-linear structures using generalized continua". In: Computer Methods in Applied Mechanics and Engineering 192.28-30 (2003). Multiscale Computational Mechanics for Materials and Structures, pp. 3233 -3244. ISSN: 0045-7825. 
[23] N.A. Fleck and J.W. Hutchinson. "A reformulation of strain gradient plasticity". In: Journal of the Mechanics and Physics of Solids 49.10 (2001), pp. 2245 -2271. ISSN: 0022-5096.

[24] J.W. Foulk. "The Singular Bar: A One-Dimensional Benchmark Problem for Computational Solid Mechanics". In: Computational Mechanics (2016).

[25] X. Gao et al. "Quantum computer aided design simulation and optimization of semiconductor quantum dots". In: Journal of Applied Physics 114 (2013), pp. 1-19.

[26] K Garikipati and TJR Hughes. "A variational multiscale approach to strain localization - formulation for multidimensional problems". English. In: COMPUTER METHODS IN APPLIED MECHANICS AND ENGINEERING 188.1-3 (2000), 39-60. ISSN: 0045-7825.

[27] P.R. Guduru, A.J. Rosakis, and G. Ravichandran. "Dynamic shear bands: an investigation using high speed optical and infrared diagnostics". In: Mechanics of Materials 33 (2001), pp. 371-402.

[28] P-A. Guidault and T. Belytschko. "On the L2 and the H1 couplings for an overlapping domain decomposition method using Lagrange multipliers". In: International Journal for Numerical Methods in Engineering 70.3 (2007), pp. 322-350. ISSN: 1097-0207.

[29] E. Gürses. "Aspects of Energy minimization in solid mechanics: evolution of inelastic microstructures and crack propagation". PhD thesis. Stuttgart, Germany: Institut für Mechanik (Banwesen), Lehrstuhl I, 2007.

[30] N. Hadjiconstantinou and A. Patera. "Heterogeneous atomistic-continuum representations for dense fluid systems”. In: International Journal of Modern Physics C 8.4 (1997), pp. 967-976.

[31] F. Han and G. Lubineau. "Coupling of nonlocal and local continuum models by the Arlequin approach". In: International Journal for Numerical Methods in Engineering 89.6 (2012), pp. 671-685. ISSN: 1097-0207.

[32] G. Hansen et al. "An MPI+X implementation of contact global search using Kokkos". In: Engineering with Computers 32.2 (2016), pp. 295-311.

[33] M. Heroux et al. An Overview of Trilinos. Tech. rep. SAND2003-2927. Sandia National Laboratories Report, Aug. 2003.

[34] V.G. Kouznetsova, M.G.D. Geers, and W.A.M. Brekelmans. "Multi-scale second-order computational homogenization of multi-phase materials: a nested finite element solution strategy". In: Computer Methods in Applied Mechanics and Engineering 193.48-51 (2004), pp. 5525 -5550. ISSN: 0045-7825.

[35] Sandia National Laboratories. Albany: A Component-Based Partial Differential Equation Code Built on Trilinos. https://github.com/gahansen/Albany. Apr. 2016.

[36] Sandia National Laboratories. Cubit. https ://cubit. sandia.gov. Feb. 2016.

[37] K. Li-Shan and D.J. Evans. "The convergence rate of the Schwarz alternating procedure (V): For more than two domains". In: International Journal for Computer Mathematics 23.3-4 (1988), pp. 295-313.

[38] P.L. Lions. "On the Schwarz alternating method I.” In: 1988, First International Symposium on Domain Decomposition methods for Partial Differential Equations, SIAM, Philadelphia.

[39] S.H. Lui. "On linear monotone iteration and Schwarz methods for nonlinear elliptic PDEs". In: Numerische Mathematik 93 (2002), pp. 109-129.

[40] T. Mathew. Domain Decomposition Methods for the Numerical Solution of Partial Differential Equations. Berlin: Springer, 2008. 
[41] S. Mikhlin. "On the Schwarz algorithm". In: Proceedings of the USSR Academy of Sciences (in Russian) 77 (1951), pp. 569-571.

[42] D. Morgenstern. "Bedründung des alternierenden Verfahrens durch orthogonalprojektion". In: ZAMM 36 (1956), pp. 7-8.

[43] A. Mota et al. "Lie-group interpolation and variational recovery for internal variables". English. In: COMPUTATIONAL MECHANICS 52.6 (Dec. 2013), 1281-1299. ISSN: 0178-7675.

[44] J. Nocedal and S.J. Wright. Numerical Optimization. New York: Springer, 1999.

[45] J. T. Ostien et al. "A 10-node composite tetrahedral finite element for solid mechanics". In: International Journal for Numerical Methods in Engineering (2016). doi : 10 .1002/nme.5218, n/a-n/a. ISSN: 1097-0207.

[46] V. Pandurangan, H. Li, and T. Ng. "A concurrent multiscale method based on the alternating Schwarz scheme for coupling atomic and continuum scaled with first-order compatibility". In: Computational Mechanics 47 (2011), pp. 1-16.

[47] M. Parks, P. Bochev, and R. Lehoucq. "Connecting atomistic-to-continuum coupling and domain decomposition”. In: Multiscale Modeling \& Simulation 7.1 (2008), pp. 362-380.

[48] M. Prager. "Schwarzuv algoritmus pro polyharmonické funkce". In: Aplikace matematiky 3.2 (1958), pp. 106-114.

[49] S. Prudhomme et al. "Computational analysis of modeling error for the coupling of particle and continuum models by the Arlequin method". In: Computer Methods in Applied Mechanics and Engineering 197.41-42 (2008). Recent Advances in Computational Study of Nanostructures, pp. 3399 -3409. ISSN: 0045-7825.

[50] A. Quarteroni. Numerical Methods for Differential Problems. Milan: Springer, 2009.

[51] H. C. Rogers. “Adiabatic plastic deformation”. In: Annual Review of Materials Science 9 (1979), pp. 283-311.

[52] J. Ryan, L. Halpern, and M. Borrel. "Domain decomposition vs. overset Chimera grid approaches for coupling CFD and CAA". In: 7th International Conference on Computational Fluid Dynamics, Big Island, Hawaii ICCFD7-1205 (July 2012).

[53] A. Salinger et al. "Albany: Using Agile Components to Develop a Flexible, Generic Multiphysics Analysis Code". In: Int. J. Multiscale Comput. Engng. (in press) (2016).

[54] F. Scarpini. "The alternating Schwarz method applied to some biharmonic variational inequalities". In: Calcolo 27.1 (1990), pp. 57-72.

[55] H. Schwarz. “Über einen Grenzübergang durch alternierendes Verfahren”. In: Vierteljahrsschriftder Naturforschenden Gesellschaft in Zurich 15 (1870), pp. 272-286.

[56] S. Slattery, P. Wilson, and R. Pawlowski. "The Data Transfer Kit: a geometric rendezvous-based tool for multiphysics data transfer". In: 2013, International Conference on Mathematics and Computational Methods Applied to Nuclear Science and Engineering, American Nuclear Society, Sun Valley, ID.

[57] B. Smith, P. Bjorstad, and W. Gropp. Domain Decomposition: parallel multilevel methods for elliptic partial differential equations. Cambridge: Cambridge University Press, 1996.

[58] S.L. Sobolev. "Schwarz's Algorithm in Elasticity Theory". In: Selected Works of S.L Sobolev. Volume I: equations of mathematical physics, computational mathematics and cubature formulats. Ed. by G.V. Demidenko and V.L. Vaskevich. New York: Springer, 2006. 
[59] W. Spotz et al. “Aeras: A Next Generation Global Atmosphere Model”. In: Procedia Computer Science 51 (2015), pp. 2097-2106.

[60] W. Sun, J. E. Andrade, and J. W. Rudnicki. "Multiscale method for characterization of porous microstructures and their impact on macroscopic effective permeability". In: International Journal for Numerical Methods in Engineering 88.12 (2011), pp. 1260-1279. ISSN: 1097-0207.

[61] W. Sun and A. Mota. "A multiscale overlapped coupling formulation for large-deformation strain localization”. English. In: Computational Mechanics 54.3 (Sept. 2014), 803-820. ISSN: 0178-7675.

[62] W. Sun, J. Ostien, and A. Salinger. "A stabilized assumed deformation gradient finite element formulation for strongly coupled poromechanical simulations at finite strain". In: International Journal for Numerical and Analytical Methods in Geomechanics 37 (2013), pp. 2755-2788.

[63] I. Tezaur et al. "Albany/FELIX: A Parallel, Scalable and Robust Finite Element Higher-Order Stokes Ice Sheet Solver Built for Advanced Analysis". In: Geoscientific Model Development 8 (2015), pp. 124.

[64] A. Toselle and O. Widlund. "Numerical Solution of Partial Differential Equations on Parallel Computers". In: Berlin: Springer, 2006. Chap. Domain Decomposition Techniques.

[65] M.-B. Tran. "Convergence properties of overlapping Schwarz domain decomposition algorithms". In: arXiv pre-print (2011).

[66] H. Tresca. "On further application of the flow of solids". In: Proceedings of the Institution of Mechanical Engineerings 30 (1878), pp. 301-345.

[67] T. Werder, J. Walther, and P. Koumoutsakos. "Hybrid atomistic-continuum method for the simulation of dense fluid flows". In: Journal of Computational Physics 205 (2005), pp. 373-390.

[68] J.A. White, R.I. Borja, and J.T. Fredrich. "Calculating the effective permeability of sandstone with multiscale lattice Boltzmann/finite element simulations”. In: Acta Geotechnica 1 (4 2006), pp. 195-209. ISSN: 1861-1125.

[69] Q. Yang, A. Mota, and M. Ortiz. "A class of variational strain-localization finite elements". In: International Journal for Numerical Methods in Engineering 62.8 (2005), pp. 1013-1037. ISSN: 1097-0207.

[70] M. Zhou, A.J. Rosakis, and G. Ravichandran. "Dynamically propagating shear bands in impact-loaded prenotched plates .1. Experimental investigations of temperature signatures and propagation speed". In: Journal of the Mechanics and Physics of Solids 44.6 (1996), pp. 981-1006. 